\title{
Hypoxia drives murine neutrophil protein scavenging to maintain central carbon metabolism
}

\author{
Emily R. Watts, ${ }^{1}$ Andrew J.M. Howden, ${ }^{2}$ Tyler Morrison, ${ }^{1}$ Pranvera Sadiku, ${ }^{1}$ Jens Hukelmann, ${ }^{2}$ Alex von Kriegsheim, ${ }^{3}$ Bart Ghesquiere, ${ }^{4}$ \\ Fiona Murphy, ${ }^{1}$ Ananda S. Mirchandani, ${ }^{1}$ Duncan C. Humphries, ${ }^{1}$ Robert Grecian, ${ }^{1}$ Eilise M. Ryan, ${ }^{1}$ Patricia Coelho, ${ }^{1}$ Gio Rodriguez Blanco, ${ }^{3}$ \\ Tracie M. Plant, ${ }^{1}$ Rebecca S. Dickinson, ${ }^{1}$ Andy Finch, ${ }^{3}$ Wesley Vermaelen, ${ }^{4}$ Doreen A. Cantrell, ${ }^{2}$ Moira K. Whyte, ${ }^{1}$ and Sarah R. Walmsley ${ }^{1}$ \\ ${ }^{1}$ University of Edinburgh Centre for Inflammation Research, Queen's Medical Research Institute, University of Edinburgh, Edinburgh, United Kingdom. ${ }^{2}$ Division of Cell Signaling and Immunology, University \\ of Dundee, Dundee, United Kingdom. ${ }^{3}$ Edinburgh Cancer Research Centre, IGMM, University of Edinburgh, Edinburgh, United Kingdom. ${ }^{4}$ Laboratory of Angiogenesis and Vascular Metabolism, Vesalius \\ Research Centre, Leuven, Belgium.
}

\begin{abstract}
Limiting dysfunctional neutrophilic inflammation while preserving effective immunity requires a better understanding of the processes that dictate neutrophil function in the tissues. Quantitative mass-spectrometry identified how inflammatory murine neutrophils regulated expression of cell surface receptors, signal transduction networks, and metabolic machinery to shape neutrophil phenotypes in response to hypoxia. Through the tracing of labeled amino acids into metabolic enzymes, proinflammatory mediators, and granule proteins, we demonstrated that ongoing protein synthesis shapes the neutrophil proteome. To maintain energy supplies in the tissues, neutrophils consumed extracellular proteins to fuel central carbon metabolism. The physiological stresses of hypoxia and hypoglycemia, characteristic of inflamed tissues, promoted this extracellular protein scavenging with activation of the lysosomal compartment, further driving exploitation of the protein-rich inflammatory milieu. This study provides a comprehensive map of neutrophil proteomes, analysis of which has led to the identification of active catabolic and anabolic pathways that enable neutrophils to sustain synthetic and effector functions in the tissues.
\end{abstract}

\section{Introduction}

The rapid recruitment of innate immune cells is critical for containing and eradicating infection and the restoration of normal tissue homeostasis. To enable effective immune responses in the tissues, leukocytes are required to adapt to the physiological challenges provided by the inflammatory niche. This is reflected in the ability of neutrophils to survive and function under conditions of limited oxygen availability (1-3). Hypoxia in the context of pneumonia is associated with poorer outcomes in patients (4, 5) and we have previously shown that in murine models of bacterial pneumonia increased sickness in hypoxia is neutrophildependent (6). Furthermore, myeloid cell-specific loss of the critical oxygen-sensing prolyl hydroxylase PHD2 resulted in a damaging inflammatory neutrophil phenotype in sterile and infective models of inflammation (7). Hypoxia is therefore an important determinant of neutrophil behavior.

Variations in both local and systemic tissue oxygenation may be amplified in disease, for example in acute respiratory distress syndrome (ARDS) and more recently in coronavirus 2019 (COVID-19)

\section{Related Commentary: https://doi.org/10.1172/JCI149495}

Conflict of interest: The authors have declared that no conflict of interest exists. Copyright: @ 2021, Watts et al. This is an open access article published under the terms of the Creative Commons Attribution 4.0 International License.

Submitted: October 7, 2019; Accepted: March 31, 2021; Published: May 17, 2021.

Reference information: / Clin Invest. 2021;131(10):e134073.

https://doi.org/10.1172/JCl134073. disease. In both contexts, worsening hypoxia and alveolar damage are associated with increased mortality (8-10). Infiltrating neutrophils in the setting of ARDS drive an overexuberant inflammatory response that is highly damaging to host tissue (8), increasing vascular injury and protein leak $(9,10)$ and promoting the alveolar epithelial injury that dictates disease progression (11-13).

The release of granule proteases at the site of injury or infection represents a key component of the neutrophil's antimicrobial repertoire and these proteases are known to contribute significantly to tissue damage in ARDS (14). To facilitate the rapid release of these proteins, granules and their contents are preformed and packaged during neutrophil maturation in the bone marrow through a temporally regulated program of transcription and translation $(15,16)$. Degranulation is necessarily a highly regulated process, as inappropriate release of these toxic proteins risks host cell and tissue damage. The ability of neutrophils to sustain damaging, proinflammatory responses in oxygen- and nutrient-limited tissues raises the important possibility that neutrophils continue to synthesize destructive granule proteases.

Here we used a lung injury model to interrogate the processes that drive harmful neutrophil phenotypes. Quantitative label-free mass-spectrometry revealed that inflammatory neutrophils have a dynamic proteome that is restructured in response to the physiological stress of hypoxia. Tracing of labeled amino acids and proteins identified the capacity of airway neutrophils to undergo de novo protein synthesis and activate lysosomal functions required to metabolize extracellular proteins to fuel this response. Consistent with this finding, systemic hypoxia aggravates lung injury, a response 
curtailed by lysosomal inhibition. This immune-metabolic leukocyte phenotype is of likely importance in both health and disease.

\section{Results}

Inflammatory neutrophils restructure their proteome in response to hypoxia. We first sought to develop a comprehensive map of the proteome of an inflammatory neutrophil to assess its capacity to respond to different environments and stimuli, something a genomics approach could not address. Moreover, the quantification of absolute protein abundance allows the unbiased analysis of the factors that differentiate the normoxic from the hypoxic neutrophil population.

Mice were exposed to nebulized lipopolysaccharide (LPS) using a sealed system to generate a uniform and reproducible lung injury. Airspace neutrophils were isolated from bronchoalveolar lavage (BAL) of mice 24 hours after induction of lung injury. Using high resolution mass spectrometry, we identified 57,000 peptides from these highly pure neutrophil preparations (Supplemental Figure 1, A and B; supplemental material available online with this article; https://doi.org/10.1172/JCI134073DS1), corresponding to more than 5000 proteins. High correlation between biological replicates was observed $\left(R^{2}>0.96\right.$, Supplemental Figure 1C). Protein copy numbers were calculated on Perseus software (17) using the proteomic ruler (18) and used to analyze the contribution of proteins to the total protein mass (Supplemental Figure 1D). Proteins were divided into quartiles by abundance (measured by label-free quantification [LFQ] intensity) and the top 2 quartiles were analyzed for KEGG pathway enrichment (as compared with the whole neutrophil proteome) using online DAVID software $(19,20)$. This analysis of the most abundant proteins highlighted that metabolic, migratory, inflammatory, and biosynthetic pathways dominate the inflammatory neutrophil proteome (Figure 1, A and B), with $7 \%$ total protein mass attributable to glycolysis, $6 \%$ to regulation of translation, and $5 \%$ to granule proteins (Figure 1, C-E). Enrichment of inflammatory, metabolic, and biosynthetic pathways was observed in neutrophils relative to the whole murine genome (Supplemental Tables 1-3). We next questioned how neutrophils restructure their proteome in response to the physiological challenge of hypoxia and low glucose availability, to which neutrophils are subjected in vivo. Given existing evidence that oxygen availability critically regulates innate immune responses to lung injury and infection $(6,7)$, we studied a model of hypoxic acute lung injury. Following nebulization (as described above), animals were transferred to a hypoxic chamber where the inhaled oxygen concentration was reduced to $10 \%$ over 1 hour. Proteomic analysis revealed a distinct protein signature in neutrophils from mice exposed to concurrent systemic hypoxia as compared with those from normoxic controls during acute lung injury (Figure $1 \mathrm{~F}$ ), with upregulation of 272 proteins and downregulation of 230 proteins $\left(P<0.05\right.$, FDR 0.05, $\mathrm{S}_{0}=0.1$; Figure $1 G)$. Hypoxic samples also showed high correlation among biological replicates (Supplemental Figure 1E).

Hypoxic neutrophils upregulate inflammatory receptors and translational machinery. Analysis of those proteins that were significantly upregulated in hypoxia revealed a number of key inflammatory receptors. Formylated peptide receptor (FPR) signaling is implicated in the regulation of neutrophil chemotaxis, degranulation, ROS production, and transcriptional regulation (21). Both FPR1 and FPR2 were significantly upregulated in the proteome of hypoxic neutrophils (Figure 2A). TNF- $\alpha$ signaling is implicated in neutrophil adhesion (22), priming (23), and apoptosis (24). TNF- $\alpha$ was detected in the BAL supernatant following LPS-induced lung injury (Supplemental Figure 2A) and hypoxic airspace neutrophils displayed higher levels of TNFRSF1b (TNF receptor 2) protein (Figure 2B). Flow cytometry confirmed higher surface expression of TNFRSF1b on hypoxic neutrophils harvested 24 hours after LPS (Supplemental Figure 2B). TNF receptor 1 was not identified on the neutrophil proteome but could be measured by flow cytometry. This identified a distinct pattern of expression with higher levels in hypoxia at 6 hours after LPS, but equivalent expression by 24 hours (Supplemental Figure 2C). Thus, hypoxia may regulate neutrophil response to TNF- $\alpha$ through dynamic regulation of surface receptor expression.

One of the most upregulated proteins in the hypoxic neutrophil proteome was the beta component of the GM-CSF receptor (CSF2RB, Figure 2C). Ligation of the GM-CSF receptor results in wide-ranging inflammatory neutrophil responses, including priming, degranulation (25), and survival (26). Flow cytometry confirmed BAL neutrophil surface expression of both the $\alpha$ and $\beta$ subunit of this heterodimeric receptor. Both subunits were upregulated in hypoxia 6 hours after LPS with equivalent surface expression between groups by 24 hours (Supplemental Figure 2, D and E). This hypoxic induction of GM-CSF receptor surface expression early in the inflammatory response is associated with increased neutrophil degranulation in vivo at 6 hours, with significantly higher levels of elastase activity (Figure 2D) and MMP9 (Figure $2 \mathrm{E}$ ) in hypoxic BAL supernatant. Degranulation is maximal at this early time point with levels of both elastase and MMP9 in the BAL falling by 24 hours (Supplemental Figure 3, A and B).

Despite detection of free elastase and MMP9 in the airways, the proteomic data set showed that airspace neutrophils retain high protein expression of azurophilic, specific and gelatinase granule proteins (Figure 2F), and showed no reduction in intracellular levels of the key granule proteins under conditions of hypoxia (Figure 2, G and H, and Supplemental Figure 3, C and D) where enhanced degranulation is observed. Persistence of granule proteins in hypoxic neutrophils despite evidence of increased degranulation led us to hypothesize that tissue neutrophils may have the capacity to synthesize granule proteins upon recruitment to the inflammatory niche, in contrast to the existing dogma that granule proteins are only synthesized and packaged in bone marrow neutrophils (15). Consistent with this possibility, hypoxia positively regulated many eukaryotic translation initiation factors (EIFs), essential for initiation of protein translation (Figure 2I) and aminoacylation of tRNAs (Figure 2, J and K). Furthermore, airspace neutrophils retain mRNAs for granule proteins (Supplemental Figure 3, E and F).

Airspace neutrophils synthesize granule proteins. To directly address the extent to which tissue neutrophils continue to synthesize inflammatory proteins, we measured the protein synthetic capacity of inflammatory BAL neutrophils by ex vivo incubation of BAL neutrophils with the heavy labeled amino acids ${ }^{13} \mathrm{C}_{6}$ and ${ }^{15} \mathrm{~N}_{4} \mathrm{~L}$-arginine and ${ }^{13} \mathrm{C}_{6}$ L-lysine. BAL neutrophils from animals that had been housed in hypoxia following LPS stimulation were used because we predicted, based on the proteomic data presented here, that these would have the greatest capacity for ongoing protein synthesis. Neutrophils were isolated 6 hours after LPS nebulization and cultured with heavy labeled amino acids for 18 hours to generate samples 


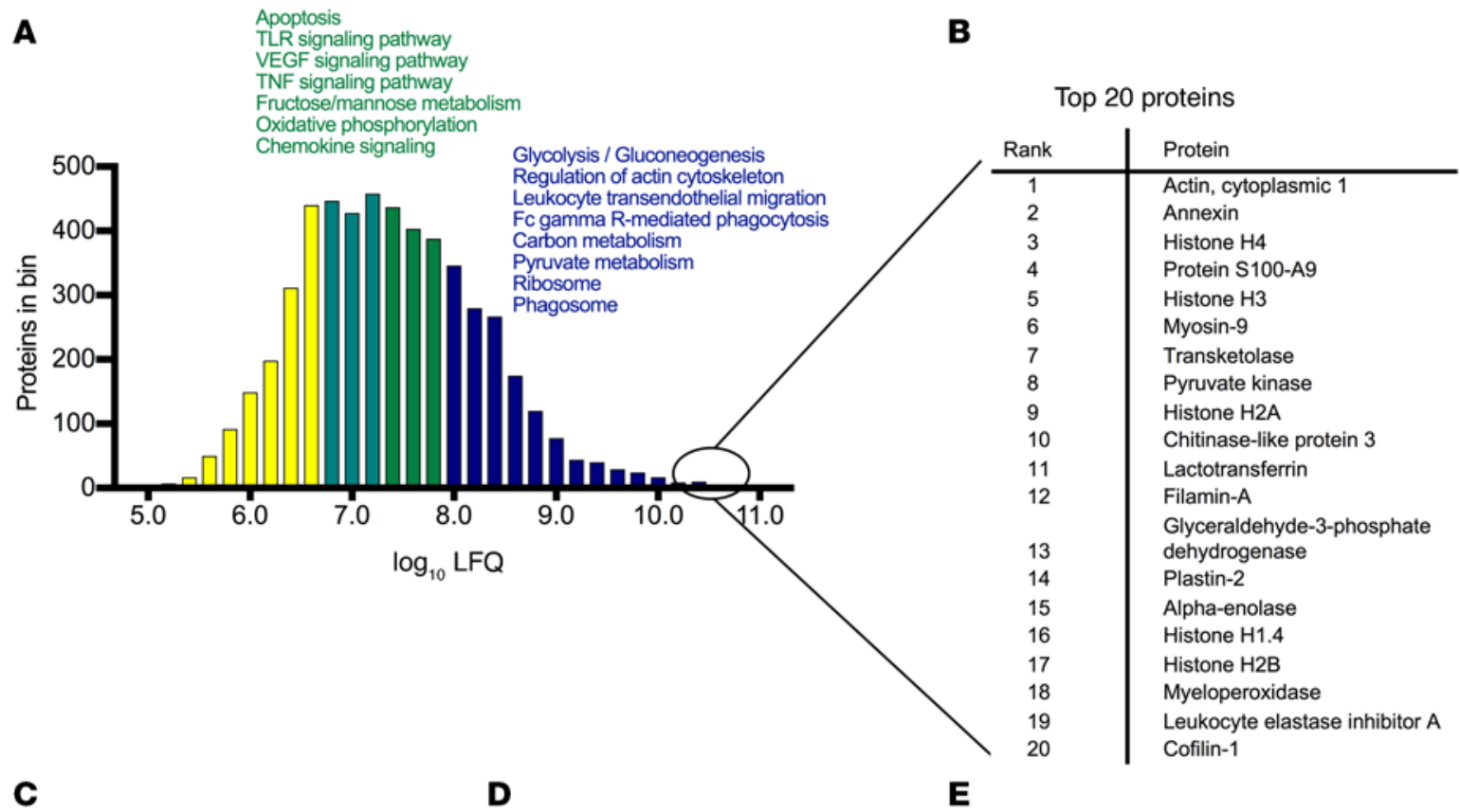

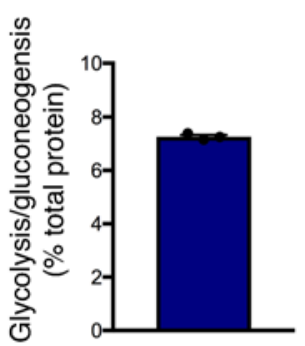

$\mathbf{F}$

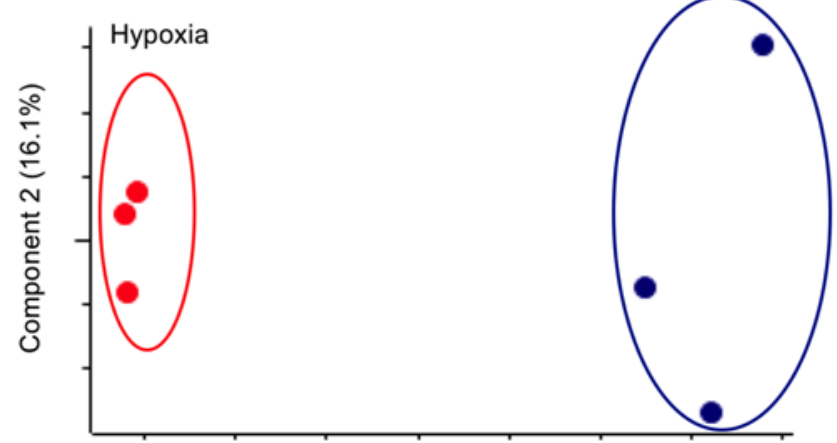

Component $1(52.7 \%)$

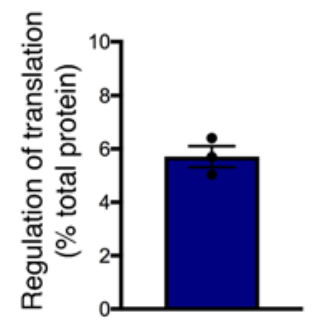

G

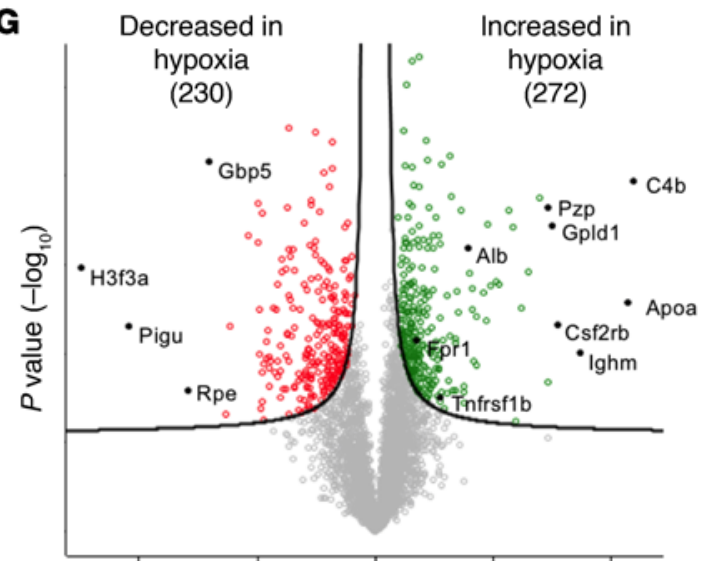

Fold change $\left(\log _{2}\right)$

Figure 1. Inflammatory neutrophils restructure their proteome in response to hypoxia. (A) Histogram of all identified proteins split into quartiles based on LFQ intensity. KEGG pathway analysis of enriched pathways in the top quartile (dark blue) and second quartile (green) are highlighted. The top 20 proteins (by LFQ intensity) are expanded in (B). The contributions of (C) the glycolysis/gluconeogenesis KEGG pathway, (D) the Gene Ontology Biological Process Regulation of Translation, and (E) granule proteins to the total protein content of a neutrophil were calculated. The proteome of normoxic and hypoxic BAL neutrophils were analyzed for (F) principle component analysis, performed without category enrichment in components. (G) Volcano plot demonstrating significant up- (green) and downregulation (red) of more than 200 proteins in hypoxic samples $\left(P<0.05, F D R 0.05, S_{0}=0.1\right)$. For all data, $n=3$ per condition. Panels $\mathbf{D}$ and $\mathbf{E}$ represent individual values and mean \pm SEM. Panel $\mathbf{F}$ represents each biological replicate. 
A

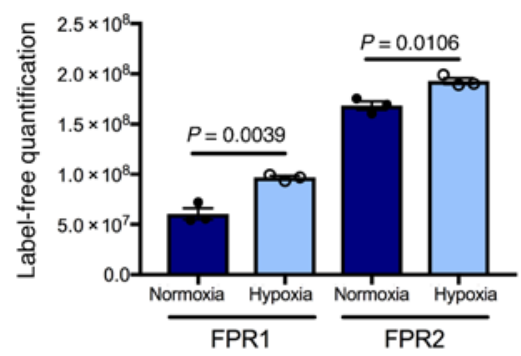

B

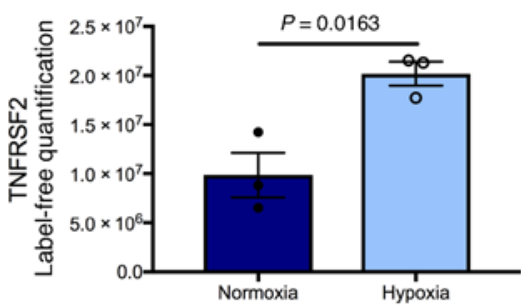

C

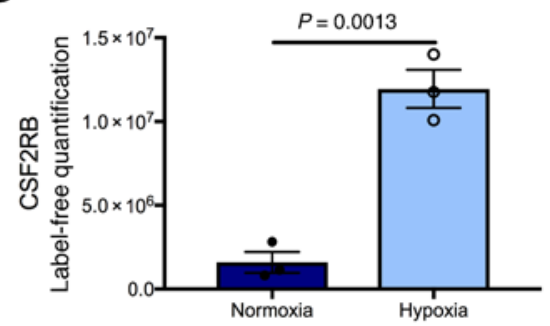

BAL supernatant

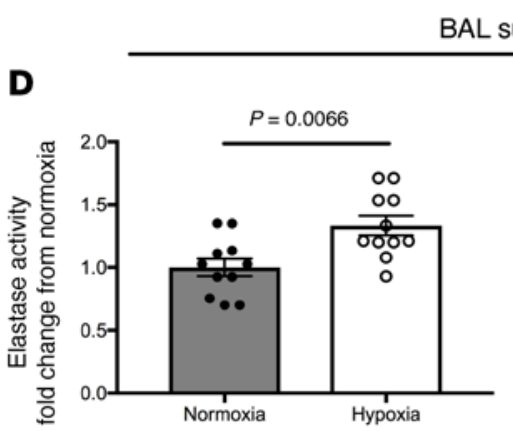

D

G
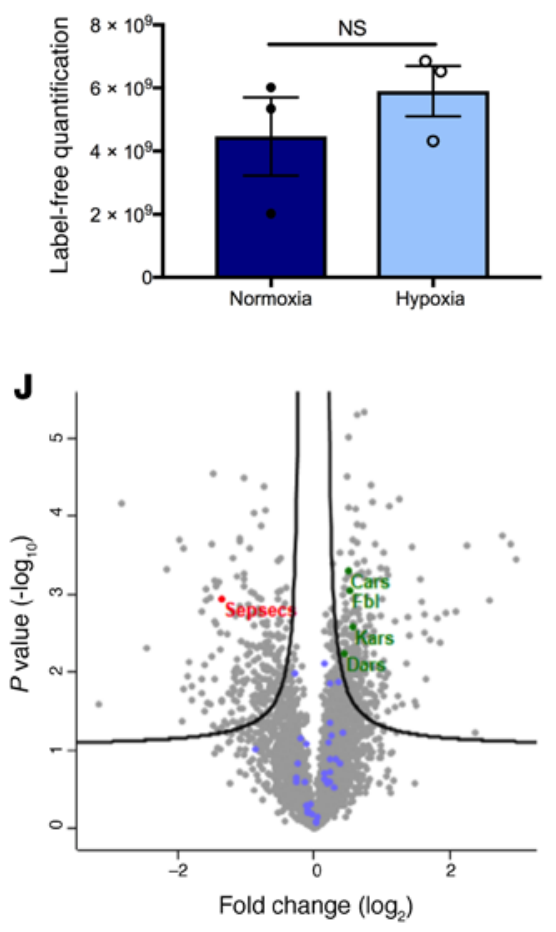
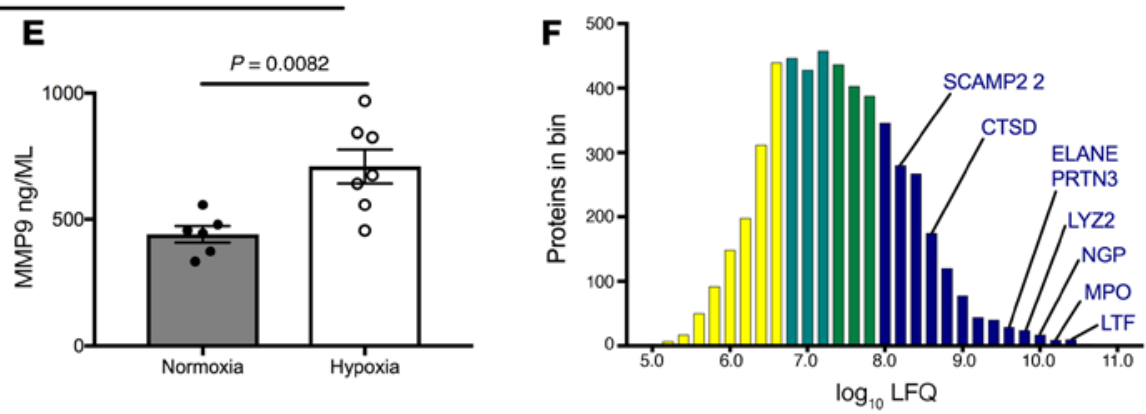

$\mathbf{H}$

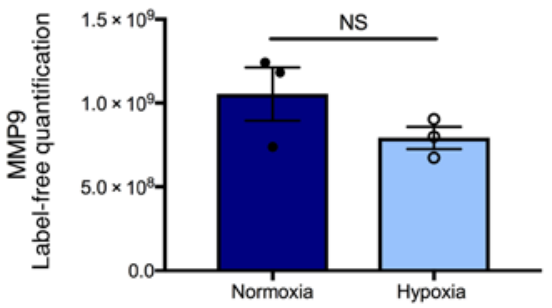

K

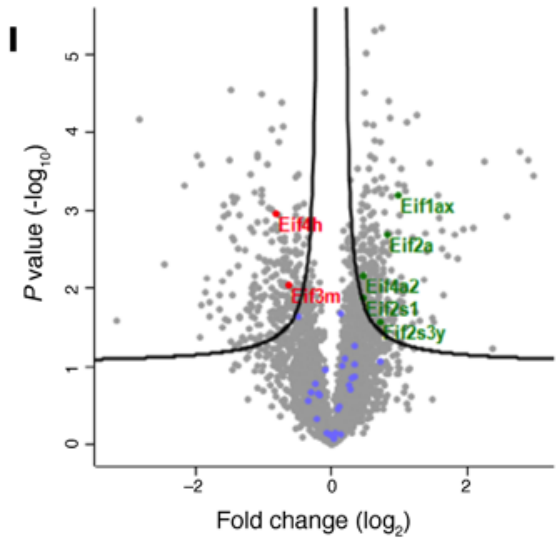

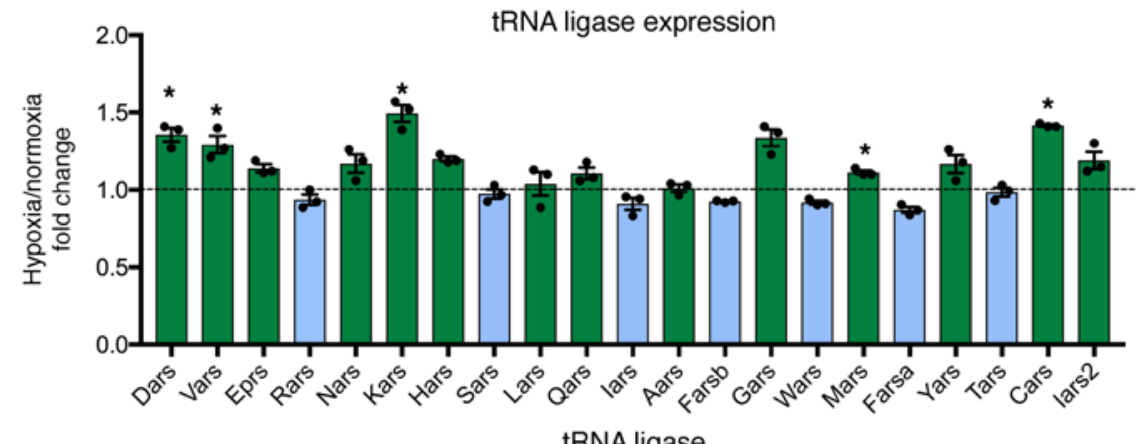

Figure 2. Hypoxia promotes a hyperinflammatory biosynthetic neutrophil phenotype. LFQ of (A) FPRs 1 and 2, (B) TNFRSF2, and (C) GM-CSF receptor subunit $\beta$ in BAL neutrophils from normoxic and hypoxic mice, $n=3$. (D) Elastase activity ( $n=11$ over 3 experiments) and (E) MMPg concentration ( $n=6$ over 2 experiments) in BAL supernatant from normoxic and hypoxic mice 6 hours after LPS, Mann-Whitney test of significance. (F) Histogram of the normoxic neutrophil proteome with the positions of granule proteins highlighted. LFQ of (C) elastase and (H) MMPg in normoxic and hypoxic BAL neutrophils, $n=3$. Volcano plots illustrating upregulation of EIFs (I) and tRNA ligases (J) with the fold change of tRNA ligases detailed in (K) with upregulated proteins highlighted in green. A-C, G, $\mathbf{H}$, and $\mathbf{K}$ used unpaired 2-tailed $t$ test of significance. In A-E, G, and $\mathbf{H}$, data represent individual values and mean \pm SEM. In K, data represent mean \pm SEM. FPR, formylated peptide receptor; TNFRSF2, TNF receptor superfamily member 2; CSF2RB, GM-CSF receptor subunit $\beta$; SCAMP2, secretory carrier-associated membrane protein 2; CTSD, cathepsin D; ELANE, neutrophil elastase; PRTN3, myeloblastin (proteinase 3); LYZ2, lysozyme C-2; NGP, neutrophil granule protein; MPO, myeloperoxidase; LTF, lactotransferrin; EIF, eukaryotic translation initiation factor. 
A

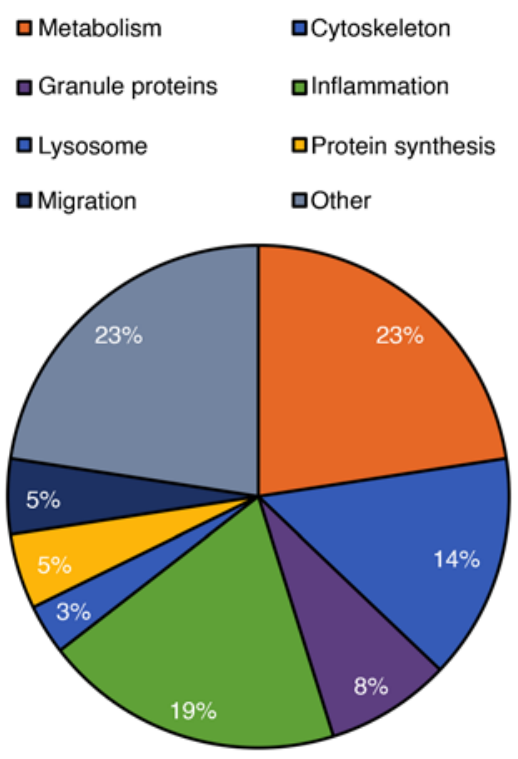

B

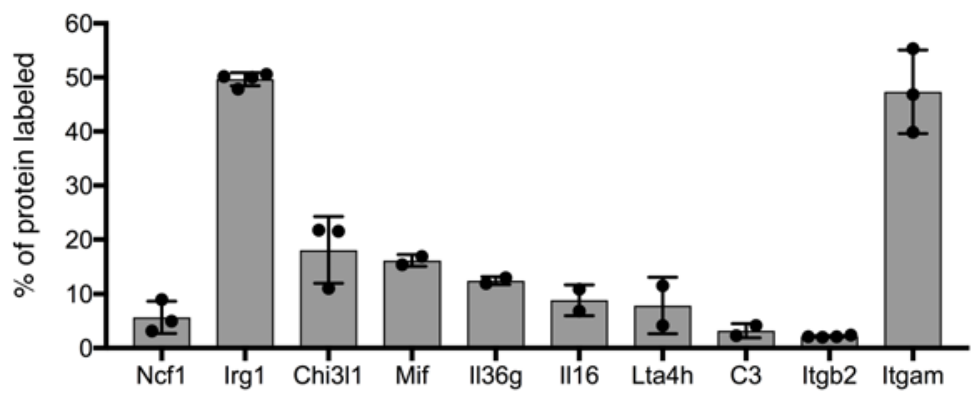

C

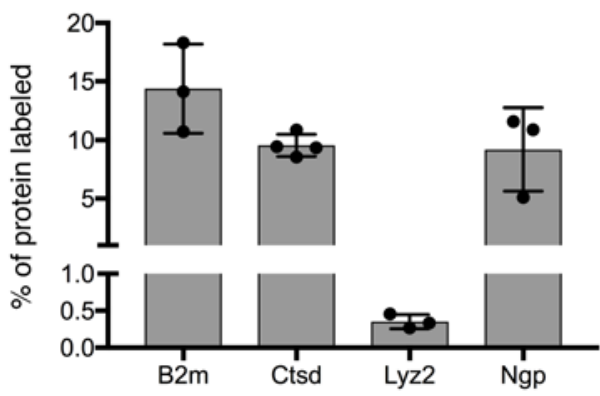

Figure 3. Airspace neutrophils synthesize granule proteins. BAL neutrophils were cultured ex vivo with labeled amino acids ( $n=4$ over 2 experiments). Labeled proteins (identified by mass spectrometry, total number $=62$ ) were assigned to a functional group as detailed in (A). Those involved in inflammation (B) and neutrophil granules (C) are detailed. Panels $\mathbf{B}$ and $\mathbf{C}$ represent individual values and mean \pm SEM with labeling expressed as the percentage of the protein that demonstrates incorporation of heavy labeled amino acid(s). Raw data are detailed in Supplemental Table 5.

equivalent to those isolated 24 hours after LPS. Sixty-two newly synthesized (labeled) proteins were identified by mass spectrometry, including abundant proteins involved in cytoskeletal regulation and metabolism (Figure 3A and Supplemental Table 5) as would be predicted from our initial proteomic screens (Figure 1, B and C). Importantly, we also identified labeling within inflammatory proteins such as complement C3 and integrins (Figure 3B) and granule proteins (Figure 3C) (27). De novo protein synthesis contributed substantially to the overall abundance of these granule proteins, with up to $10 \%$ heavy label incorporation. Coupled with the evidence of preserved intracellular granule proteins despite enhanced degranulation, these data are consistent with de novo synthesis of inflammatory mediators, including granule proteins by tissue neutrophils, providing a mechanism by which hypoxic neutrophils would be predicted to drive a hyperinflammatory immune response.

Neutrophils scavenge extracellular proteins for fuel. Despite their well-recognized reliance on glycolysis for ATP production, we found that airway neutrophils are subject to marked hypoglycemia. Only very low levels of glucose were present in BAL supernatant (Supplemental Figure $4 A)(28,29)$. In order to more accurately determine the absolute concentration of glucose that cells within the airways are exposed to (without the diluting effect of the BAL), we also performed LCMS analysis of airway surface liquid that was adsorbed onto filter cards directly from the upper airways. This confirmed low levels of glucose in the airways of both normoxic and hypoxic mice (Figure 4A). Although hypoxic neutrophils did upregulate the glucose transporter Glut 3 (Figure $4 \mathrm{~B}$ ), this did not translate to an increase in intracellular glucose-6-phosphate (Figure 4C), likely due to the low substrate availability. We therefore asked how airway neutrophils are able to support the observed synthetic function in this metabolite deplete environment. In contrast to intracellular glucose levels, hypoxic neutrophils were found to have significantly higher levels of intracellular glutamine and glutamate (Figure 4D). LCMS analysis of airway fluid did detect glutamine, but there was no increase in hypoxic airways (Figure $4 \mathrm{E}$ ). Furthermore, at a protein level, neutrophils did not reproducibly express the important SLC-38 family of glutamine transporters (Supplemental Figure 4B) (30), suggesting that transport of free glutamine does not account for the observed increase in intracellular glutamine in hypoxia.

Survey of the neutrophil proteome led to the surprising observation that neutrophils contain the extracellular proteins albumin, $\operatorname{IgM}$, and fibronectin, and that they increase in abundance when neutrophils are exposed to hypoxic lung injury (Figure 4, F-H). This led us to question whether neutrophils can scavenge proteins to use as an alternative source of amino acids, a mechanism employed by tumor cell lines to meet their increasing metabolic demands (31-33). Consistent with glutamine being an important metabolic substrate (34), a number of key enzymes and transporters involved in glutaminolysis (glutaminase, glutamate dehydrogenase, alpha ketoglutarate dehydrogenase, phosphoenolpyruvate carboxykinase 2 , and the dicarboxylate transporter) are upregulated at the protein level in hypoxia (Figure $5 \mathrm{~A}$ ). The ability of neutrophils to use an active protein uptake pathway to fuel their energetic requirements at sites of inflammation would represent an advantageous metabolic adaptation to the inflamed lung niche.

To definitively address whether neutrophils can use extracellular proteins for anaplerosis, we fed ex vivo airspace neutrophils with ${ }^{13} \mathrm{C}_{5}$ glutamine-labeled proteins and traced the presence of heavy carbons into their metabolic intermediaries. Label could be traced into TCA cycle metabolites including citrate, fumarate, and 
A

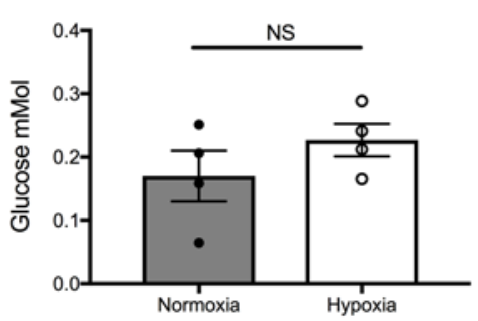

D

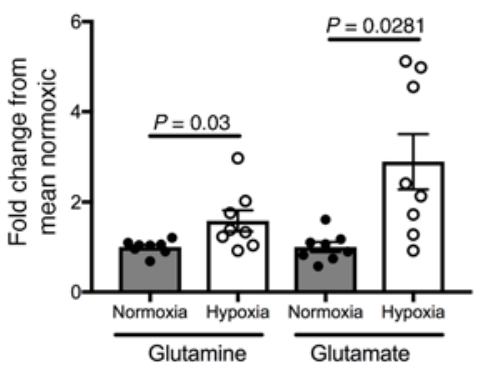

B

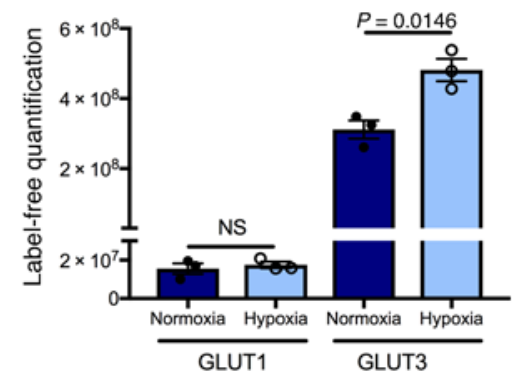

E

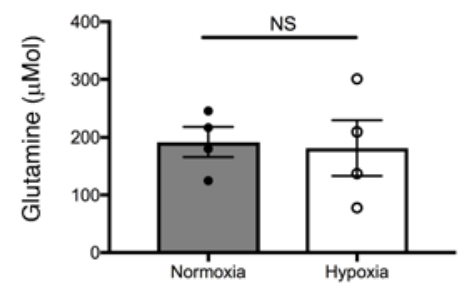

C

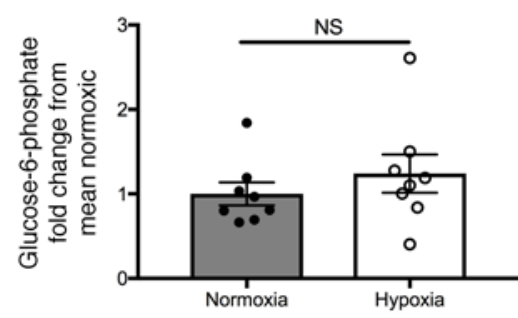

Extracellular protein uptake
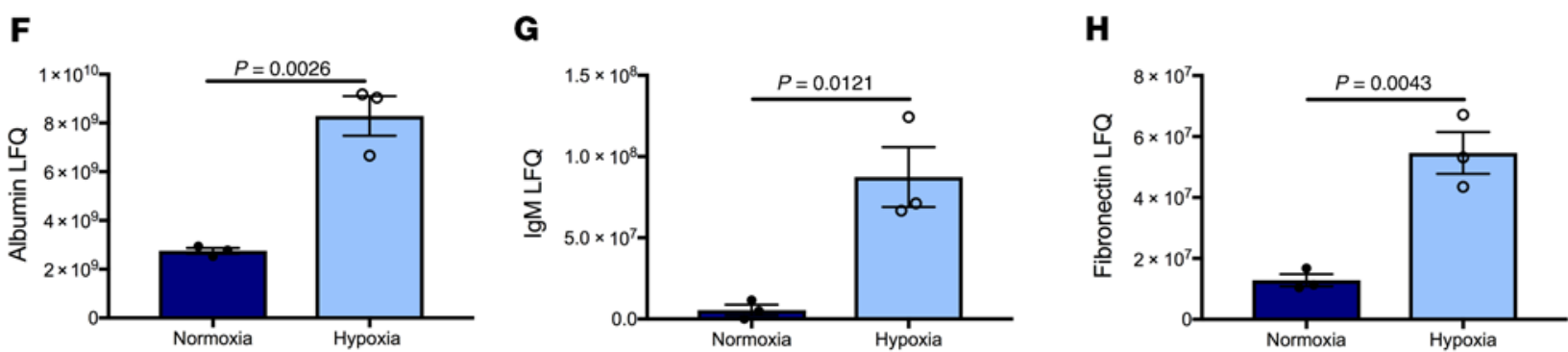

Figure 4. Hypoglycemic neutrophils scavenge extracellular proteins. (A) Glucose concentration of airway surface liquid ( $n=4$ over one experiment). (B) LFQ of glucose transporters GLUT1 and GLUT3, $n=3$. (C) Intracellular abundance of glucose-6-phosphate measured by HPLC-MS ( $n=8$ over 2 experiments). (D) Intracellular abundance of glutamate and glutamine ( $n=8$ over 2 experiments). (E) Glutamine concentration of airway surface liquid from normoxic and hypoxic mice 24 hours after LPS ( $n=4$ over one experiment). LFQ of (F) albumin, (C) IgM, and (H) fibronectin in BAL neutrophils, $n=3$. A and C-E used a Mann-Whitney test of significance. B and F-H used the unpaired 2-tailed $t$ test of significance. All data are from normoxic and hypoxic mice 24 hours after nebulized LPS.

malate (Figure 5, B-D, and Supplemental Figure 4, C-F), consistent with flux of protein-derived glutamine into central carbon metabolism. We did not identify any evidence of reductive carboxylation, with no $\mathrm{m}+5$ citrate (Supplemental Figure $4 \mathrm{C}$ ) and no $\mathrm{m}+3$ malate (Supplemental Figure 4D) identified. These experiments did not allow us to conclusively comment on TCA cycling, and though we did identify the presence of small amounts of $m+2$ citrate, we did not see labeling within oxaloacetate (data not shown). Neutrophils therefore have the capacity to use extracellular proteins as an amino acid source for metabolic intermediaries.

Hypoxia and $m T O R C 1$ activity regulate neutrophil catabolism of scavenged extracellular proteins. To investigate the use of extracellular proteins by neutrophils, we used Texas red-labeled bovine serum albumin (BSA) to measure protein uptake and double-quenched Green-labeled (DQ-Green-labeled) BSA (which requires proteolytic cleavage to emit green fluorescence) to measure breakdown. We used human blood neutrophils in addition to murine BAL neutrophils to confirm that protein scavenging is of functional relevance in a broader range of neutrophil subsets. Incubation of healthy human peripheral blood neutrophils with Texas red BSA demonstrates rapid uptake with a significant increase in Texas red signal after a 30-minute incubation (Figure 6A). Rapid proteolysis also occurs with a significant increase in the DQ-Green signal observed after 90 minutes (Figure 6A). To confirm that the observed DQ-Green signal was a direct result of intracellular proteolysis, rather than extracellular proteolysis and uptake of unquenched fluorescent BSA, the DQ-Green BSA time course was repeated following a wash at 30 minutes to remove residual extracellular albumin. Consistent with intracellular breakdown of DQ-Green BSA, there was a significant increase in intracellular fluorescence over a 2-hour incubation period with fresh media (Figure 6B).

In order to delineate the mechanism by which neutrophils take up albumin, we used confocal microscopy to assess localization of FITC-BSA and Texas red $70 \mathrm{kDa}$ dextran, a substance predominantly taken up via macropinocytosis (35). Merged images revealed partial colocalization of the BSA signal with the $70 \mathrm{kDa}$ dextran (Figure 6C), indicating that one route of albumin uptake is via macropinocytosis (36). 
A

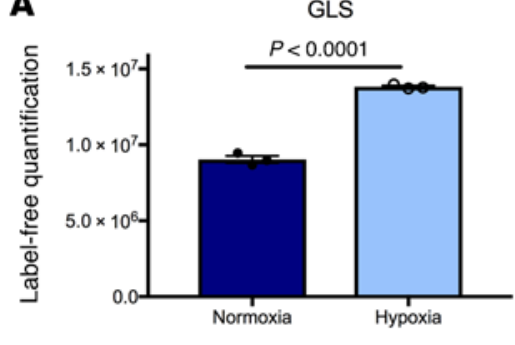

GLUD1
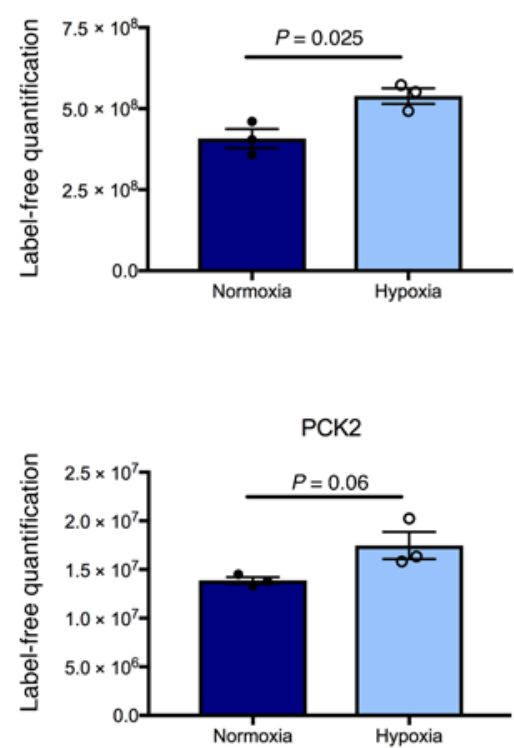

B

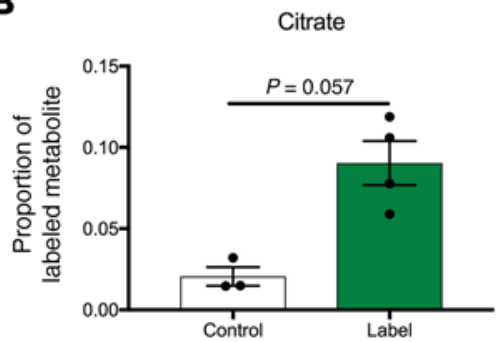

Glutaminolysis

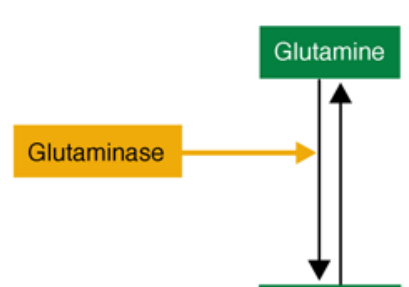

Glutamate
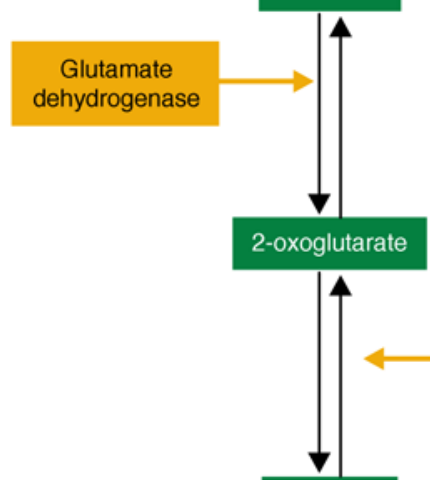

Succinate

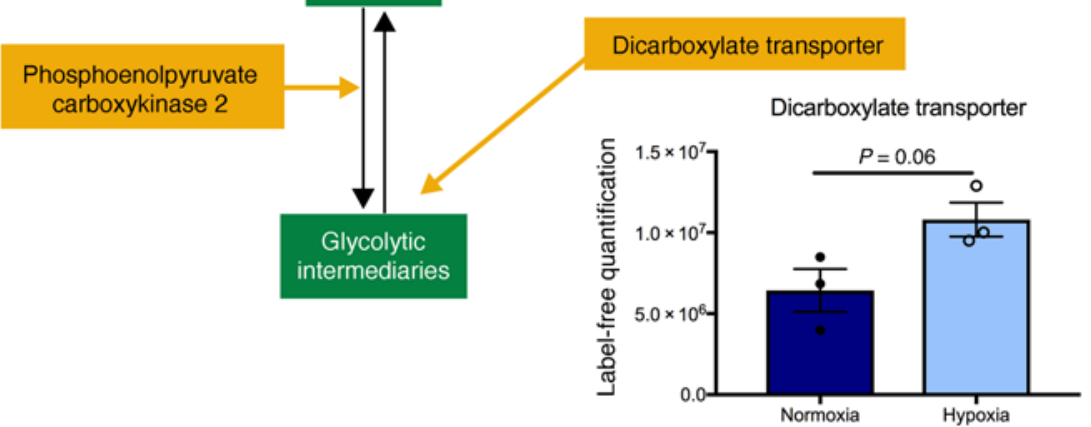

C

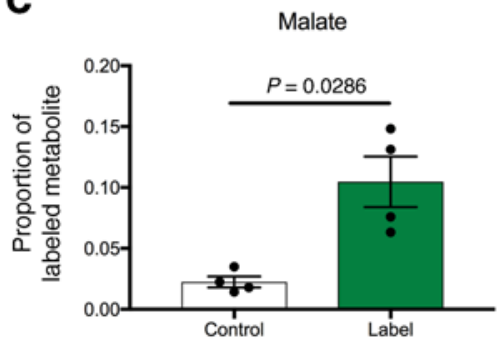

OGDH

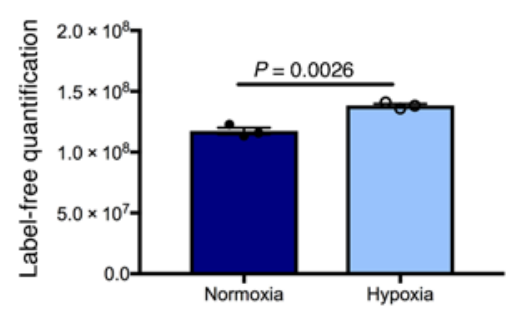

$\alpha-K G$

dehydrogenase

D Fumarate

Figure 5. Glutamine from scavenged proteins fuels hypoxic neutrophils. (A) LFQ of proteins involved in glutaminolysis, $n=3$, unpaired 2-tailed $t$ test of significance. (B-D) Highly pure BAL neutrophils harvested 6 hours after nebulized LPS from hypoxic mice were cultured ex vivo for 18 hours in hypoxia $\left(1 \% \mathrm{O}_{2}\right)$ in glucose-free media supplemented ${ }^{13} C_{5}$ glutamine-labeled (green) or unlabeled (white) HEK cell lysates. Metabolite abundance and labeling was measured by HPLC-MS ( $n=4$ over 2 experiments), Mann-Whitney test of significance. Diagram represents the glutaminolysis pathway with enzymes in yellow and metabolic intermediaries in green. GLS, glutaminase; GLUD1, glutamate dehydrogenase 1; OGDH, 2-oxoglutarate dehydrogenase; PCK2, phosphoenolpyruvate carboxykinase.

We hypothesized that in neutrophils, nutrient availability may play a role in regulating protein uptake. In light of the glucosedeplete nature of the inflamed airway, we incubated LPS-treated human peripheral blood neutrophils in the presence or absence of glucose and compared normoxic $\left(21 \% \mathrm{O}_{2}\right)$ with hypoxic $\left(1 \% \mathrm{O}_{2}\right)$ culture. Comparing the signal from neutrophils incubated with Texas red BSA with those incubated with DQ-Green BSA identified an interesting distinction between conditions that favor uptake versus those that favor breakdown. In glucose-replete media, hypoxia enhances albumin uptake. This trend is preserved in glucose depletion but is nonsignificant (Figure 6D). In both normoxia and hypoxia, the absence of glucose results in reduced uptake of albumin but this is significant only in hypoxia (Figure 6D). In contrast, hypoxia enhances albumin breakdown in both glucose-replete and -deplete conditions (Figure 6E), and glucose depletion does not limit albumin breakdown. This suggests that the increase in albumin catabolism that is driven by hypoxia is sufficient to overcome any reduced substrate uptake due to glucose deprivation. This is illustrated by 
A

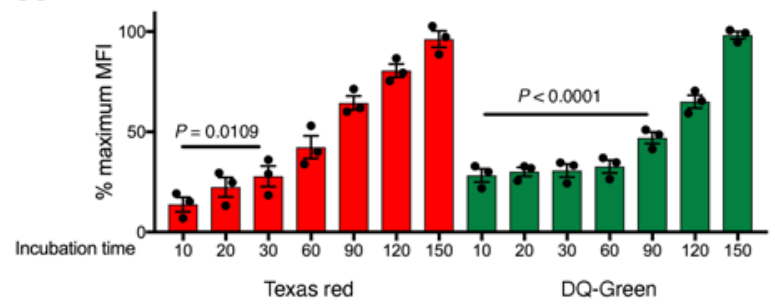

C

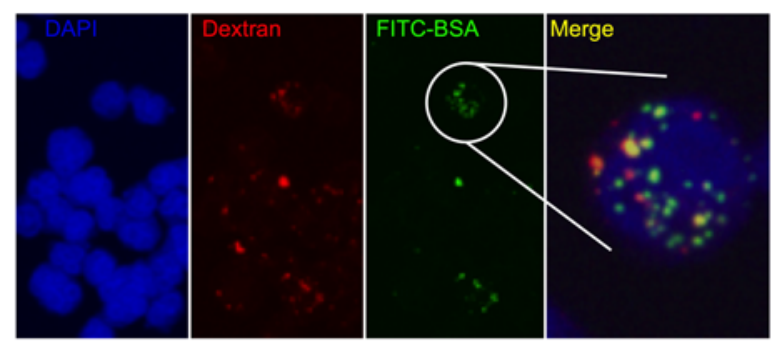

E

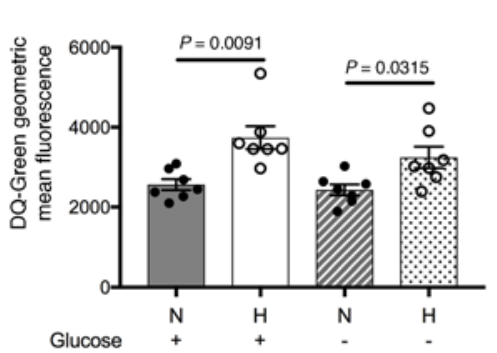

H

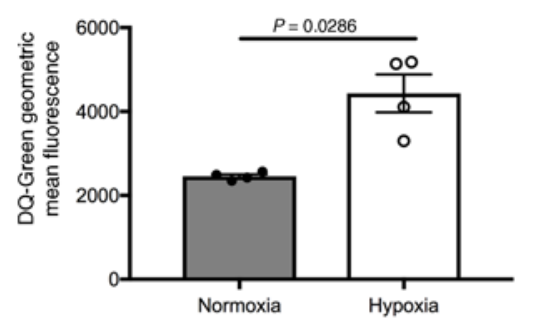

$\mathbf{F}$

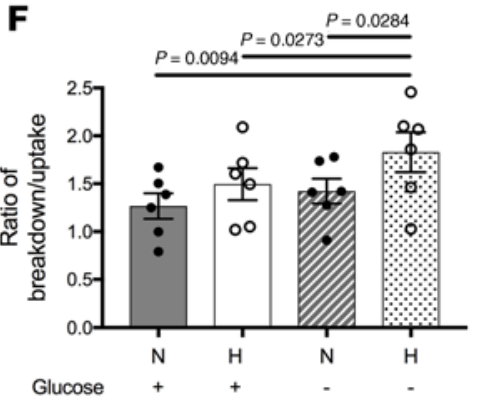

I

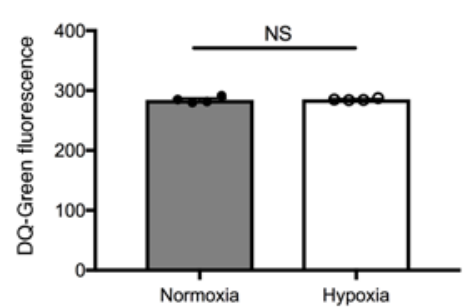

B

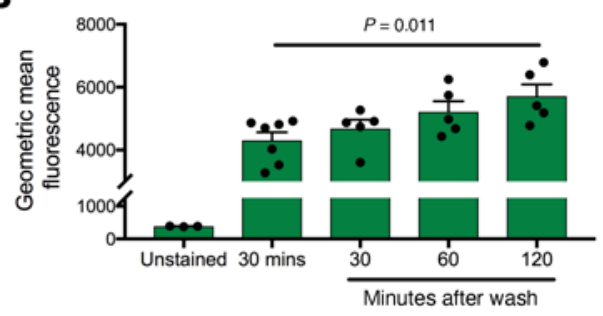

D

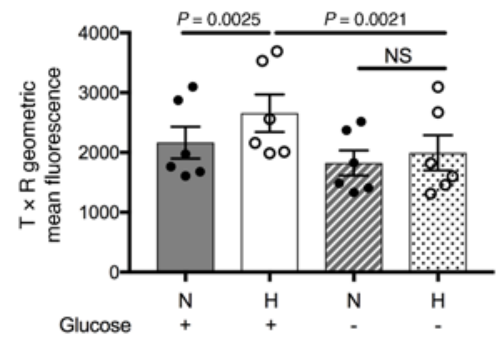

G

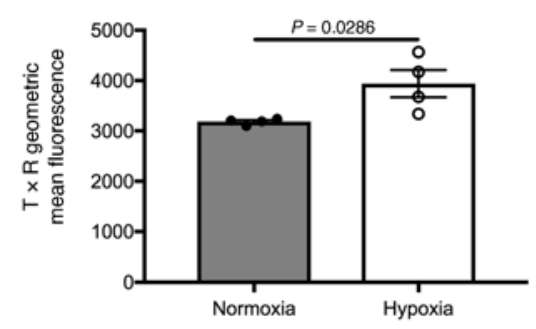

$\mathbf{L}$

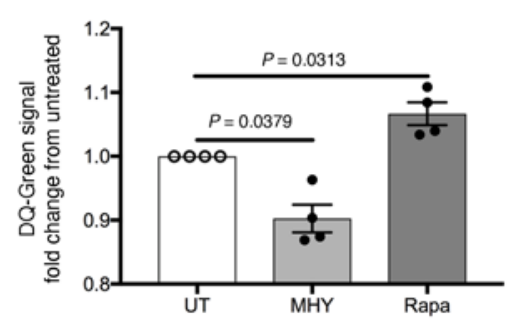


Figure 6. Hypoxia promotes mTOR regulated neutrophil catabolism of extra-cellular albumin. Time course of human peripheral blood neutrophils incubated with (A) Texas red-labeled BSA or DQ-Green BSA for 150 minutes (data expressed as \% maximum signal) and (B) DQ-Green BSA for 30 minutes followed by washing and then further incubation for 120 minutes. $\mathbf{A}$ and $\mathbf{B}$ were measured by flow cytometry ( $n=3$ over 2 experiments). (C) Confocal microscopy of human peripheral blood neutrophils incubated with FITC-BSA (green) and $70 \mathrm{kDa}$ dextran (red) with DAPI nuclear staining in blue and red/green colocalization highlighted in yellow. Geometric mean fluorescence of LPS-treated human neutrophils incubated with (D) Texas red-labeled BSA $(n=6)$ or (E) DQ-Green BSA ( $n=7$ over 3 experiments) in normoxia or hypoxia $\left(1 \% \mathrm{O}_{2}\right)$ in RPMI with or without glucose $(11 \mathrm{mM})$. (F) Ratio of DQ-Green to Texas red signal demonstrating breakdown efficiency in the conditions described in $\mathbf{D}$ and $\mathbf{E} ; n=6$ over 3 experiments. Geometric mean fluorescence of BAL neutrophils from normoxic or hypoxic mice 24 hours after LPS incubated ex vivo in the corresponding oxygen tension with (C) Texas red BSA or (H) DQ-Green BSA ( $n=4$ over one experiment). (I) Fluorescence of cell culture supernatant from H. mTORC1 activity (proportion of phosphorylated S6 kinase) in (J) LPS-treated human peripheral blood neutrophils cultured in normoxia or hypoxia $\left(1 \% \mathrm{O}_{2}\right)$ with or without glucose (11 mM) ( $n=7$ over 4 experiments) and (K) murine BAL neutrophils isolated 24 hours after nebulized LPS (mice housed in normoxia or hypoxia); $n=8$ over 2 experiments. Representative blots shown. (L) Geometric mean fluorescence of LPS-treated human neutrophils incubated with DQ-Green BSA and pretreated with MHY1485 $(2 \mu \mathrm{M})$ or Rapamycin (50 $\mathrm{nM}) ; n=4$ over 2 experiments. $\operatorname{In} \mathbf{A}$ and $\mathbf{B}$, data represent mean $\pm \mathrm{SEM}$. In D-J, data represent individual values and mean \pm SEM. A, B, D-F, J, and $\mathbf{L}$ were analyzed by ordinary 1-way ANOVA with multiple comparisons. G-I and $\mathbf{K}$ used the Mann-Whitney test of significance.

the ratio of DQ-Green to Texas red fluorescence in each condition, which can be used as an indicator of the proteolytic activity of the cells. This showed that LPS-stimulated neutrophils incubated in hypoxia without glucose (conditions most closely resembling the hypoxic airway) had significantly higher proteolytic capacity than in any other condition (Figure 6F). This is consistent with the hypothesis that in conditions of nutrient scarcity, neutrophils exploit their environment to scavenge alternative energy supplies in the form of protein, and that hypoxia positively regulates this process. In order to investigate the in vivo relevance of this observation, inflammatory BAL neutrophils from normoxic and hypoxic mice were cultured ex vivo with Texas red BSA and DQ-Green BSA (with those from normoxic mice cultured in $21 \%$ oxygen and those from hypoxic mice cultured in $1 \%$ oxygen). In these ex vivo cells, hypoxia drives both increased protein uptake and degradation (Figure 6, G and $\mathrm{H})$. Thus, transmigration into the alveolar space and/or exposure to the inflammatory milieu further augments neutrophil protein scavenging capacity. Measurement of cell culture supernatant fluorescence confirmed that the enhanced DQ-Green signal in hypoxia did not represent enhanced extracellular DQ activation secondary to increased degranulation of neutrophil proteases (Figure 6I).

In tumor cells, the mammalian target of rapamycin (mTOR) and its associated complexes mTORC1 and mTORC2 are essential in integrating signals regarding nutrient availability. Downstream signaling then determines the cellular response to these signals in terms of transcription and translation of new proteins and in directing proliferation (32). Interestingly, data in fibroblasts showed that mTORC1 activity negatively regulated lysosomal degradation of proteins taken up from the environment (32). We speculated that there might also be an inverse correlation between
mTORC1 activity and protein breakdown by neutrophils. Our proteomic data confirmed that neutrophils express the key components of mTORC1, mTORC2, and the Ragulator complex proteins LAMTOR1-3 required for translocation of mTORC to the lysosomal membrane (Supplemental Figure 5, A and B) (37). The basal activity of mTORC1 in human peripheral blood neutrophils was analyzed using the phosphorylation status of the downstream target $\mathrm{S} 6$ kinase (S6K). In unstimulated neutrophils, mTORC1 activity was low and LPS treatment resulted in a significant increase in phosphorylated S6K (Supplemental Figure 5C). Oxygen and glucose availability dramatically altered the activity of mTORC1 in LPS-treated human peripheral blood neutrophils with suppression of mTORC1 activity by both hypoxia and glucose deprivation (Figure 6J), an effect replicated in vivo with diminished expression of phosphorylated S6K in airspace neutrophils isolated from hypoxic mice (Figure 6K). To investigate whether mTORC activity directly regulates catabolism of extracellular proteins, human peripheral blood neutrophils were incubated with the mTORC inhibitor Rapamycin or with the mTORC activator MHY1485 (38) prior to the addition of LPS and DQ-Green BSA. With mTORC inhibition, we observed a significant increase in proteolysis (as measured by DQ-Green fluorescence) and with mTORC activation, a significant decrease (Figure 6L). Thus, mTORC activity inhibits breakdown of proteins in neutrophils, and mTORC suppression under oxygen- and glucose-deplete conditions relieves this inhibition.

Hypoxia upregulates lysosomal catabolism of extracellular protein by tissue neutrophils for anaplerosis. The lysosome is essential for cellular protein degradation. To investigate the importance of lysosomal trafficking for extracellular protein catabolism by neutrophils, we first verified the cellular localization of scavenged albumin. Using confocal microscopy, we observed colocalization of DQ-Green BSA with the lysosomal marker LAMP1 (Figure 7A). Thus, neutrophils have capacity to traffic proteins from the extracellular environment to the lysosome. KEGG pathway analysis of those proteins upregulated by hypoxia at a proteomic level revealed enrichment for the lysosome pathway (fold enrichment 1.564, $P=$ 0.032), with more detailed analysis of known lysosomal proteins (39) confirming the majority of lysosomal pathway members to be upregulated by hypoxia (Figure 7B and Supplemental Table 4). In the hypoxic inflamed airway, neutrophils can upregulate both the lysosomal pathway (Figure 7B) and the enzymes required to metabolize the resultant free amino acids, such as glutamine (Figure $5 \mathrm{~A}$ ). The importance of the lysosome for protein catabolism is further supported by the observation that a lysosomal protease inhibitor E64 (32) (Figure 7C) and an inhibitor of lysosomal acidification chloroquine (40) (Figure 7D) diminish the ability of neutrophils to breakdown DQ-Green BSA in vitro. Furthermore, lysosomal proteins accounted for $14 \%$ of newly synthesized proteins in the previous amino acid tracing experiments (Figure $3 \mathrm{~A}$ ), further highlighting the importance of this organelle in the inflammatory neutrophil. Neutrophils therefore have the capacity to regulate uptake and degradation of extracellular proteins via the lysosome to provide amino acids required for the provision of metabolic intermediaries. Hypoxia and glucose deprivation positively regulate these processes via mTOR and the lysosome.

Hypoxia promotes a damaging hyperinflammatory neutrophil phenotype in vivo. In response to systemic hypoxia, we have observed 
A

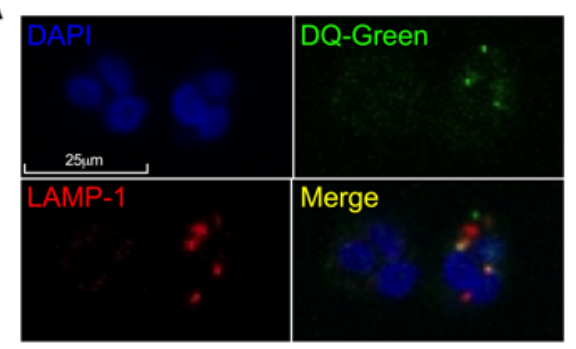

C
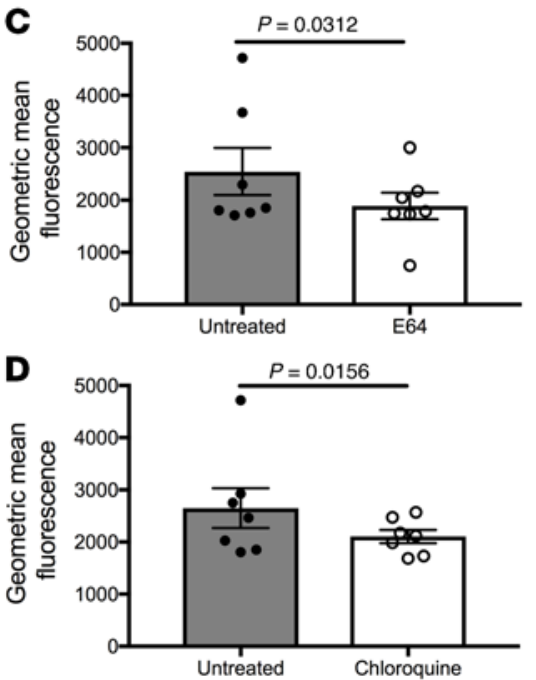

B

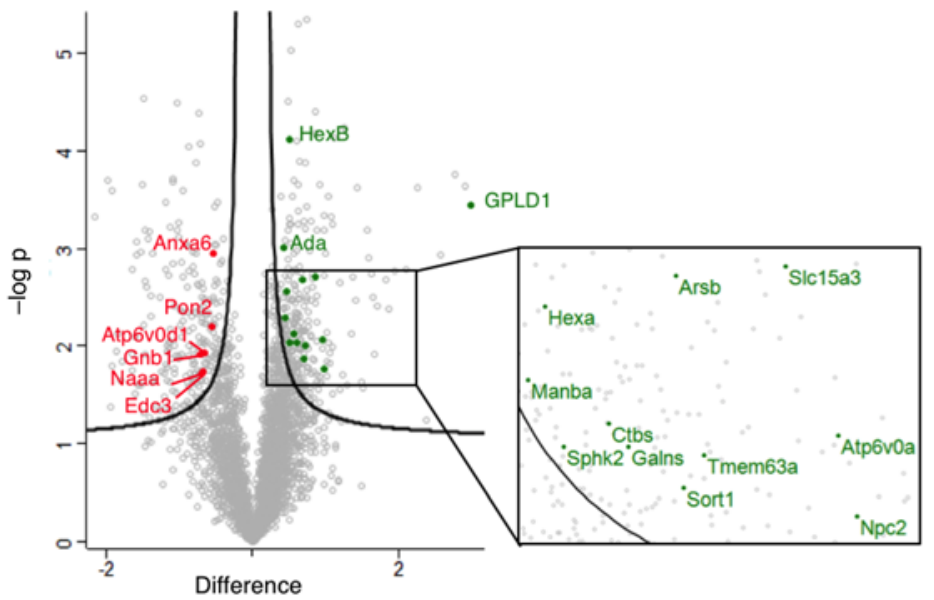

Figure 7. Lysosomal catabolism of extracellular protein provides substrate for de novo protein synthesis. (A) Confocal microscopy of human peripheral blood neutrophils incubated with DQ-Green BSA (green) and subsequently fixed and permeabilized and stained for the lysosomal marker LAMP-1 (red) with DAPI nuclear staining in blue and red/green colocalization highlighted in yellow. (B) Volcano plot comparing the proteomes of hypoxic and normoxic neutrophils (as in Figure $1 \mathrm{H}$ ) with lysosomal pathway members which are significantly up- (green) and downregulated (red) highlighted. Geometric mean fluorescence of LPS-treated human neutrophils incubated with DQ-Green BSA and pretreated with (C) protease inhibitor E64 or (D) the inhibitor of lysosomal acidification chloroquine; $n=7$ over 4 experiments, Wilcoxon 2 -tailed test of statistical significance. Panels $\mathbf{C}$ and $\mathbf{D}$ represent individual values and mean $\pm \mathrm{SEM}$. that neutrophils restructure their proteome and enhance extracellular protein scavenging. This is associated with increased inflammation within the tissues. To determine the biological consequences of these proteomic adaptations, we explored the physiological outcomes of exposing mice to systemic hypoxia in the context of acute lung injury. Concurrent exposure of mice to LPS and hypoxia resulted in profound hypothermia over the first 24 hours (Figure 8A), a response replicated in the setting of bacterial pneumonia (6) and in keeping with a more severe inflammatory response. In keeping with hypoxic upregulation of inflammatory receptors and degranulation (Figure 2, A-E), analysis of the BAL fluid supernatant revealed an increase in lung injury demonstrated by significantly more capillary leak of albumin (Figure 8B) and IgM (Figure 8C) in hypoxia. The very high levels of intracellular albumin and IgM observed in hypoxia (Figure 4, F and G) are therefore likely to be a consequence of both increased substrate availability and increased uptake. While we did demonstrate increased capacity for protein uptake in hypoxic BAL neutrophils (Figure 6G), this relatively modest increase is unlikely to be sufficient to overcome the nearly 3-fold increase in BAL albumin concentration in the lungs of hypoxic mice. The excess lung damage was independent of neutrophil number, with preserved BAL (Figure 8D) and interstitial (Figure 8E) neutrophil counts, despite lower circulating neutrophil numbers (Figure 8F) in hypoxia. Evidence of increased sickness and more significant lung injury in response to hypoxia, together with in vivo evidence of activated neutrophil surface phenotypes confirm important biological consequences of the observed proteomic changes (Figure $8, \mathrm{G}$ and $\mathrm{H})(41,42)$. In hypoxia, increased protein leak provides abundant substrate for the upregulated processes of protein scavenging and catabolism. De novo protein synthesis contributes to ongoing inflammatory mediator production (Figure 3) and, in hypoxia, increased granule protease release into the lung is associated with increased lung injury, culminating in a vicious cycle of persistent inflammation. To confirm the physiological relevance of lysosomal protein trafficking for inflammation outcomes, we explored the consequence of in vivo administration of chloroquine in the LPS lung injury model. Administration of i.p. chloroquine $(43,44)$ resulted in a partial rescue of the hypothermia observed following LPS challenge (Figure 8I), without a reduction in neutrophil numbers (Figure 8J). When BAL neutrophils were cultured ex vivo with DQ-Green albumin for 2 hours, as predicted from our in vitro treatment of human blood neutrophils, there was an associated reduction in DQ-Green fluorescence in airway neutrophils harvested from the chloroquine-treated mice, indicating that i.p. chloroquine is sufficient to reduce the lysosomal breakdown capacity of neutrophils recruited to the airways (Figure 8K). Moreover, analysis of the BAL supernatant showed a suppression of neutrophil granule protease production with a reduction in elastase activity in the airways of chloroquine-treated mice (Figure 8L). Thus, systemic chloroquine treatment in mice leads to reduced neutrophil lysosomal activity and is associated with diminished release of neutrophil granule proteins in the airways. Further work will be required to delineate whether the observed effects of chloroquine relate directly to lysosomal inhibition and whether specific targeting of the lysosome can drive resolution of neutrophil-mediated inflammatory lung disease.

\section{Discussion}

The factors that tip the balance of neutrophil phenotype from functional immunity to pathological inflammation remain poorly 

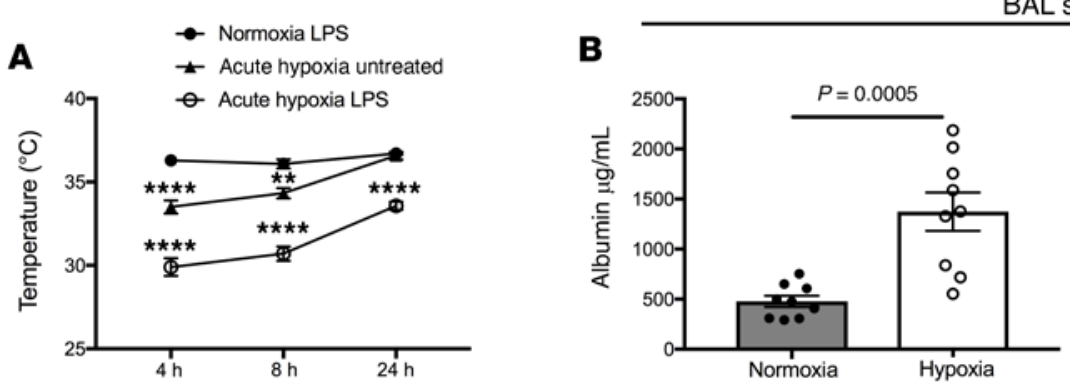

BAL supernatant

Neutrophil counts
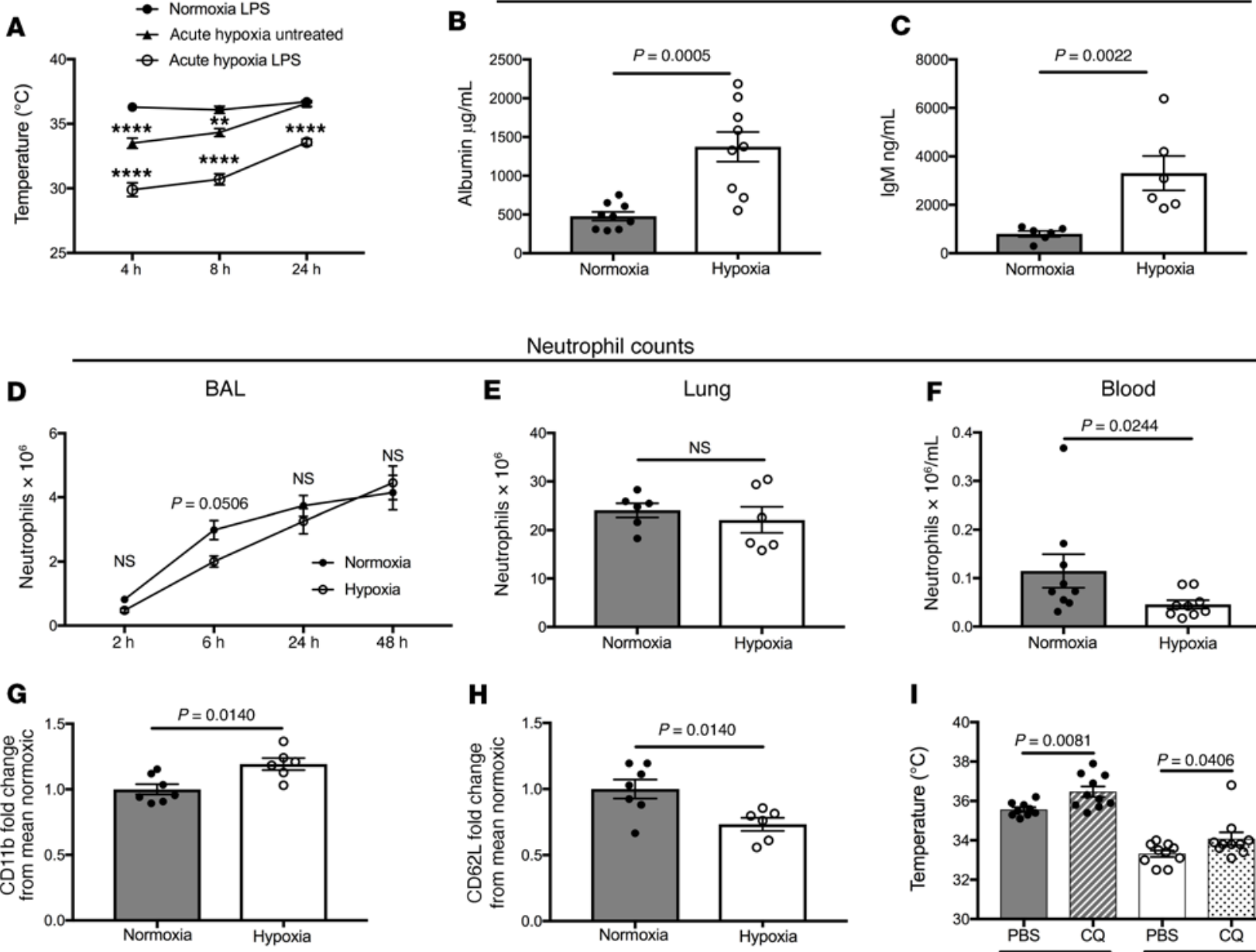

J
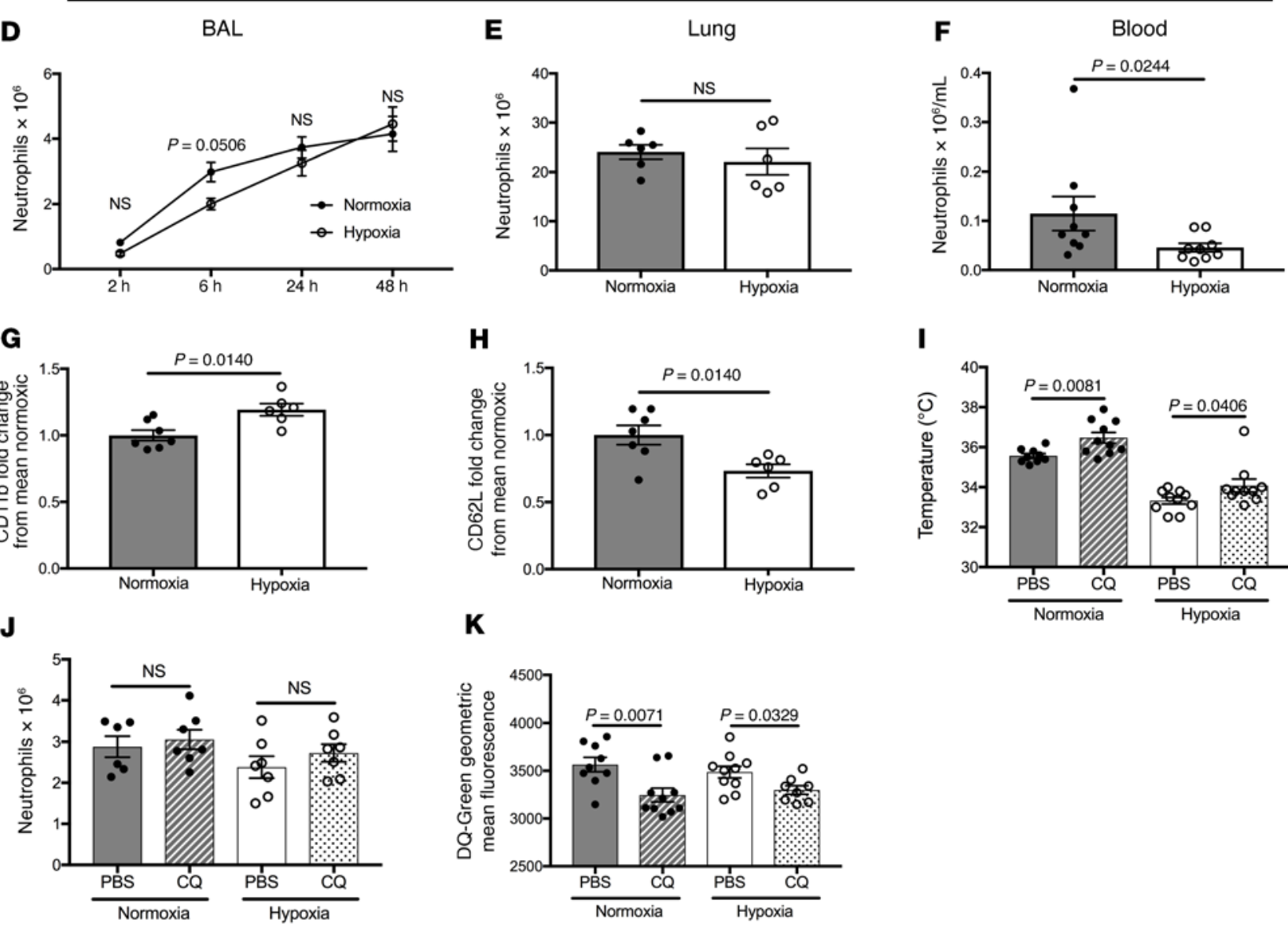

L
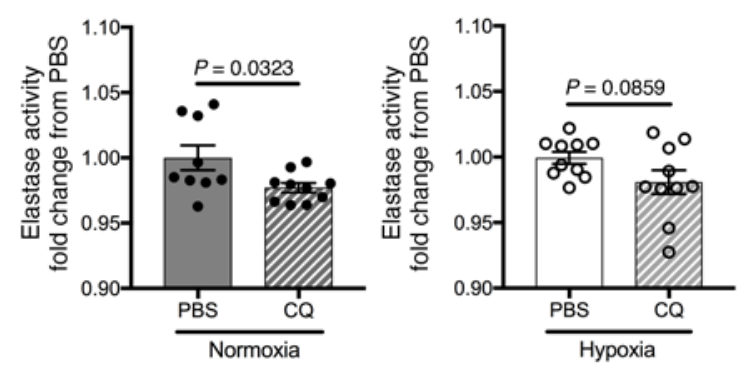
Figure 8. Hypoxia promotes a hyperinflammatory neutrophil phenotype abrogated by lysosomal inhibition. (A) Body temperature of mice housed in hypoxia $\left(10 \% \mathrm{O}_{2}\right)$ or normoxia for 24 hours after nebulized LPS and untreated mice housed in hypoxia; $n=4$ over 2 experiments, analyzed by 2-way ANOVA with Tukey's multiple comparisons test. ${ }^{* *} P<0.01,{ }^{* * *} P<$ 0.0001 . BAL supernatant from normoxic and hypoxic mice 24 hours after LPS was analyzed for (B) albumin ( $n=9$ over 2 experiments) and (C) IgM ( $n$ $=5$ over one experiment) by ELISA. (D) BAL neutrophil counts from normoxic or hypoxic mice following nebulized LPS; $n=4$ (one experiment) at 2 hour and 48 hour time points, or $n=8$ ( 2 experiments) at 6 hour and 24 hour time points. Significance analyzed by multiple $t$ tests (corrected for multiple comparisons using Bonferroni method). (E) Lung neutrophil counts ( $n=$ 6 over 2 experiments) and (F) blood neutrophil counts ( $n=9$ over 3 experiments) of mice housed in normoxia or hypoxia for 24 hours following LPS nebulization. BAL neutrophils isolated 24 hours after nebulized LPS from normoxic or hypoxic mice were analyzed by flow cytometry for expression of (C) CD11b and (H) CD62L ( $n=6-7$ over 2 experiments). (I) Body temperatures and (J) BAL neutrophil counts from mice 24 hours after LPS nebulization, treated with i.p. chloroquine or i.p. PBS at 4 hours and housed in normoxia or hypoxia. (K) BAL neutrophils incubated ex vivo with DQ-Green BSA and analyzed by flow cytometry and (L) elastase activity in BAL supernatant from the same mice. I, $\mathbf{K}$, and $\mathbf{L}: n=9-10$ over 3 experiments. J: $n=6-7$ over 2 experiments. J-L used unpaired $t$ test. I used Mann-Whitney test of significance (data not normally distributed). In $\mathbf{A}$ and $\mathbf{D}$, data represent mean \pm SEM. Data in B, C, and E-L represent individual values and mean \pm SEM.

understood. As a consequence, designing therapies for the many pathologies that are known to be mediated by neutrophilic inflammation remains a major challenge. Study of the proteome of the inflammatory neutrophil provides insights into the importance of biosynthesis for neutrophil function, with neutrophils demonstrating enrichment for the ribosome and RNA transport as well as inflammatory pathways. Comparison of normoxic and hypoxic neutrophil proteomes demonstrated that neutrophils have the capacity to restructure their proteomes to adapt to physiological stressors and regulate these important biosynthetic functions. Exploring the biological consequence of these metabolic adaptations provided insights into the mechanisms by which neutrophils sustain their effector functions in the tissues.

Protein-rich exudates are pathognomonic of acute lung injury. In this work, we observed that protein leak is not only a marker of lung damage but also has the capacity to drive further inflammation, with the finding that airspace neutrophils can take up extracellular proteins. We showed for the first time that neutrophils not only scavenge proteins from their environment but use these ingested proteins to contribute amino acids to maintain central carbon metabolism. Moreover, this response is quick, with lysosomal breakdown of extracellular albumin occurring within 90 minutes and evidence of contribution to metabolite synthesis at 18 hours. This is consequently of biological importance given the predicted lifespan of tissue neutrophils following recruitment during an acute inflammatory response $(45,46)$, which is further extended by the presence of both tissue and systemic hypoxia $(1,7)$.

The hypoxic inflamed lung represents an optimal niche to increase neutrophil capacity for protein consumption, providing increased access to extracellular albumin and IgM, suppression of mTORC activity, and promotion of lysosomal catabolism. We propose that initial hypoxic induction of formylated peptide, TNF- $\alpha$, and GM-CSF inflammatory receptors further provides a mechanism by which early neutrophil mediated damage and protein leak would provide substrate to fuel this continued inflammatory response.

The observation that, in hypoxia, high concentrations of granule proteases in the supernatants are not accompanied by reduced intracellular levels of these proteins led us to investigate whether tissue neutrophils continue to synthesize granule contents. Incorporation of labeled amino acids into newly synthesized granule proteins confirmed their ongoing production by tissue neutrophils. We also provide direct evidence that neutrophils can release amino acids from scavenged proteins, fueling central carbon metabolism. These observations, in conjunction with our findings of increased levels of granule proteins in hypoxia, where protein scavenging is also increased, raise the possibility that amino acids from scavenged proteins may also provide substrate for protein synthesis. The enhanced capacity for protein translation observed in hypoxic neutrophils, coupled with increased protein substrate availability, would support this concept. This hypothesis is further supported by the evidence that in vivo inhibition of lysosomal protein catabolism led to reduced neutrophil elastase production by airway neutrophils. Further work is required to confirm whether amino acids from scavenged proteins can be incorporated in newly synthesized proteins by neutrophils in the inflammatory niche.

There are clear parallels to be drawn between the harsh oxygen- and glucose-limited environment of the inflamed lung and the tumor microenvironment (47). Macropinocytosis of protein has been shown to be an important metabolic adaptation by tumor cells to metabolic stress $(32,33)$. In human blood neutrophils, the observed colocalization of FITC-BSA with dextran and LAMP1 also highlights a role for macropinocytosis in the delivery of extracellular proteins to the neutrophil lysosome. It is unlikely that macropinocytosis reflects the only mechanism of protein uptake given the partial correlation of BSA with dextran and the capacity of neutrophils, as professional phagocytes, to ingest particles by a number of alternative routes.

The importance of extracellular protein uptake to the metabolic adaptations of neutrophils within the inflammatory niche was explored in a series of labeled protein tracing experiments. Our data provide direct evidence that immune cells are able to derive amino acids from proteins for central carbon metabolism. This substrate utilization is promoted by hypoxic upregulation of the lysosomal compartment and concurrent suppression of mTORC1 activity, augmented by glucose deprivation, allowing unfettered catabolism of proteins in the lysosome. Increased expression of enzymes involved in glutaminolysis further supports metabolism of the resulting free amino acid pool in hypoxia facilitating anaplerosis. Neutrophils use gluconeogenesis to generate glycogen stores with glycogen cycling retaining energy production when extracellular glucose is limited (34). In this work, we propose that glutamine derived from scavenged proteins feeds into this gluconeogenic response, generating ATP. It is interesting to speculate whether substrate-level phosphorylation at the level of succinate coA ligase could also be contributing to ATP production in this context. The inflammatory capability of neutrophils is not therefore limited to releasing proteases from preformed granules, but is dynamic and adapted to the inflammatory niche. Understanding the factors that regulate the capacity of neutrophils to synthesize and release granule proteins will be a significant focus moving forward. Direct evidence of proteinderived amino acid incorporation into newly synthesized proteins 
has proved technically challenging but remains an area of active investigation. It is also interesting to speculate whether microenvironmental factors, including hypoxia and metabolic stress may shape the transcriptome of the neutrophil, determining the program of protein synthesis, and whether the mechanism of release of these newly synthesized proteins is by further rounds of degranulation or involves alternative routes of exocytosis. This work has focused on the breakdown of extracellular proteins but it is important to acknowledge that the closely related process of autophagy, whereby cells recycle intracellular proteins, may also contribute to the amino acid pool in the neutrophil. In future work, it will be important to consider how the processes of transmigration and activation themselves alter the neutrophil proteome.

Given the frequency with which other inflamed tissues (48), including the bowel (49) and joint (50), are found to be hypoxic, the pathways identified here are likely to be relevant more broadly in the regulation of neutrophilic inflammation. These data contribute to our understanding of the mechanisms by which neutrophils sense and adapt to local microenvironmental stresses, characteristic of the inflammatory niche and, as such, provide new strategies for the treatment of neutrophil-mediated inflammatory disease.

\section{Methods}

\section{Isolation and culture of human peripheral blood neutrophils}

Human peripheral blood neutrophils were isolated from sodium citrate anticoagulated blood by dextran sedimentation and discontinuous Percoll as previously described (51). Purity was assessed by morphology on cytocentrifuge slides.

\section{Murine LPS-induced acute lung injury}

Mice were treated with LPS from Pseudomonas aeruginosa $10(1 \mathrm{mg} / \mathrm{mL}$, Sigma Aldrich) for 10 minutes using oxygen driven nebulization into a custom-built sealed system to generate a uniform and reproducible acute lung injury $(7,52)$. Following LPS treatment, animals were housed in either normal room air or in hypoxia. Animals were culled using an overdose of i.p. anesthetic at the appropriate time point (2-48 hours after nebulization) and tissues harvested.

\section{Exposure to hypoxia}

Mice were exposed to hypoxia $\left(10 \%\right.$ inspired $\left.\mathrm{O}_{2}\right)$ in an InVivo Hypoxic Cabinet System (Coy Labs) with excess $\mathrm{CO}_{2}$ scavenged using Sofnolime soda lime chips (Molecular Products) with color indicator. In models of acute hypoxia, LPS-stimulated, or naive mice were housed in hypoxia for 2 to 48 hours prior to tissue harvest.

\section{Intraperitoneal chloroquine administration}

Following administration of nebulized LPS, as described above, mice were housed in hypoxia or normoxia and at 4 hours were treated with i.p. chloroquine diphosphate (Sigma-Aldrich) at a dose of $50 \mathrm{mg} / \mathrm{kg}$ diluted in PBS. Control mice were given i.p. PBS alone. Following chloroquine or PBS injection, animals were housed as before in either normoxia or hypoxia until being culled by overdose of i.p. anesthetic 24 hours after LPS.

\section{Murine neutrophil isolation}

BAL neutrophils. The trachea was cannulated using a $24 \mathrm{G}$ cannula, which was tied in place. The lungs were washed with $4 \mathrm{~mL}$ ice cold
$0.9 \% \mathrm{NaCl}$ and BAL fluid kept on ice until further processing. Cell counts were carried out using a hemocytometer and purity assessed by morphology on cytospin.

A discontinuous Percoll gradient (78\%, 69\%, and 52\% Percoll) was used to purify BAL neutrophils for RNA, protein, and metabolite assays. Residual red blood cells were lysed by hypotonic saline lysis. Purity was assessed by morphology and flow cytometry with neutrophil purity greater than $98 \%$.

\section{Murine lung digest}

Following the BAL protocol described above, the right ventricle was cannulated, and the circulation flushed with $10 \mathrm{~mL}$ of ice-cold PBS to minimize circulating leucocytes within the lung vasculature prior to lung harvest. The lung tissue was mechanically dissociated using scissors prior to enzymatic digestion with collagenase V $(0.8 \mathrm{mg} / \mathrm{mL}$, Sigma-Aldrich), Collagenase D (0.625 mg/mL, Roche), Dispase $(1 \mathrm{mg} / \mathrm{mL}$, Gibco) and DNase (30 $\mu \mathrm{g} / \mathrm{mL}$, Roche) made up in RPMI. The digest was then passed through a $100 \mu \mathrm{m}$ filter, washed with PBS and red cell lysis performed (Sigma-Aldrich) prior to resuspension in FACS buffer (PBS with $5 \%$ BSA and $2 \mathrm{mM}$ EDTA). The cell suspension was passed through a $40 \mu \mathrm{m}$ filter, counted using a NucleoCounter automatic cell counter (Eppendorf) and processed for flow cytometry analysis.

\section{Cell culture}

For all experiments, murine neutrophils were cultured at a density of $1 \times$ $10^{6} / \mathrm{mL}$ and human neutrophils at a density of $5 \times 10^{6} / \mathrm{mL}$. Media were supplemented with $1 \%$ penicillin streptomycin (Gibco) and 10\% dialyzed FCS (Gibco) unless otherwise stated. Neutrophils were cultured in RPMI 1640 media with $2 \mathrm{mM}$ glutamine (Gibco). The glucose-replete media had $11 \mathrm{mM}$ D-glucose. Standard cell culture was carried out in a Thermo Fisher Scientific Heraeus $\mathrm{BB} 15 \mathrm{CO}_{2}$ incubator. For hypoxic cell culture, conditions were as above but cells were cultured in a Ruskin hypoxic work station (Ruskin) with an $\mathrm{O}_{2}$ of $1 \%$ and $\mathrm{CO}_{2}$ of $5 \%$. Humidity in the hypoxic work station was maintained at $70 \%$.

HEK cell lysates were used as a substrate to analyze the downstream use of scavenged proteins. HEK 293 cells were a gift from Alex Von Kriegsheim (Institute of Genetics and Molecular Medicine, University of Edinburgh, Edinburgh, United Kingdom). Control cells were cultured in glucose-replete DMEM (Gibco) with 10\% dialyzed FCS, $1 \%$ penicillin streptomycin, and $2 \mathrm{mM}$ L-glutamine. For glutamine labeling experiments, standard L-glutamine was substituted with ${ }^{13} \mathrm{C}_{5}$-labeled glutamine (Cambridge Isotopes) for 72 hours prior to being harvested and lysed.

\section{Acetone precipitation of HEK cell proteins}

Cells were harvested and washed 3 times with ice-cold PBS. The cell pellet was then lysed by hypotonic lysis in $4 \mathrm{~mL}$ of sterile water prior to sonication and acetone precipitation, with supernatants (containing small molecules such as metabolites and free amino acids) discarded. Samples were centrifuged at $15,000 \mathrm{~g}$ for 10 minutes to remove insoluble protein material and supernatants used for downstream culture. Protein content was measured by Pierce BCA assay (Thermo Fisher Scientific) and normalized between labeled and unlabeled samples.

\section{Ex vivo culture of BAL neutrophils with glutamine-labeled HEK cell proteins}

BAL neutrophils were isolated and purified by Percoll gradient as detailed above. Following purification, neutrophils were resuspended 
in glucose- and glutamine-free DMEM (Gibco) supplemented with 10\% FCS and $400 \mu \mathrm{g} / \mathrm{mL}$ HEK cell protein isolated by acetone precipitation as above. Neutrophils were cultured at a density of $1 \times 10^{6} / \mathrm{mL}$ for 18 hours in hypoxia. Following this period of culture, cells were washed 3 times with sterile $0.9 \% \mathrm{NaCl}$ prior to methanol lysis as detailed below.

\section{HPLC-MS analysis of metabolites}

Highly pure BAL neutrophils were analyzed for intracellular metabolite abundance either immediately following isolation or following culture with labeled HEK lysates. Following Percoll purification as described, cells were washed in ice-cold $0.9 \% \mathrm{NaCl}$ prior to resuspension in $80 \%$ Methanol. Following centrifugation at 20,000 $g$ for 10 minutes at $4^{\circ} \mathrm{C}$, pellets were retained and protein content measured by Pierce BCA assay (Thermo Fisher Scientific). Samples were analyzed at the VIB Center for Cancer Biology in Leuven using a Dionex UltiMate 3000 LC System (Thermo Fisher Scientific) coupled to a Q Exactive Orbitrap mass spectrometer (Thermo Fisher Scientific) operated in negative mode. Data collection was performed using Xcalibur software (Thermo Fisher Scientific). All data values were subsequently corrected for protein content based on the BCA assay.

Airway surface liquid was analyzed by insertion of custom made filter cards (Hunt Developments) into the upper airway of mice prior to BAL. Metabolites were subsequently eluted from the filter cards in $200 \mu \mathrm{L}$ of 40:40:20 methanol/acetonitrile/water. Following extraction, samples were stored at $-80^{\circ} \mathrm{C}$. Relative metabolite abundance was determined using ion-pairing reverse phase high performance liquid chromatography (RP-HPLC) or hydrophilic interaction liquid chromatography coupled to a Q-Exactive Orbitrap Mass Spectrometer. Data were analyzed in a targeted manner using Xcalibur (Thermo Fisher Scientific) against an in-house compound library to integrate the area under the curve at the expected retention time and the average of 2 replicates subjected to analysis. In order to extrapolate absolute values for airway glucose and glutamine, a standard curve was generated. The filter cards from the in vivo experiments were weighed to ascertain the mean volume of airway surface fluid that had been adsorbed onto the card $(6.4 \mu \mathrm{L})$. An equivalent volume of either glucose or glutamine was applied to filter cards at varying concentrations and these samples were eluted and run in parallel with the experimental samples.

\section{Sample preparation for proteomic analysis}

Percoll purified BAL neutrophils were lysed in SDS lysis buffer with tris(2-carboxyethyl)phosphine at a $10 \mathrm{mM}$ working concentration and boiled for 5 minutes. Samples underwent 15 minutes of sonication then incubation with benzonase for 15 minutes at $37^{\circ} \mathrm{C}$. Protein concentration was estimated using the EZQprotein quantitation kit (Invitrogen). Following quantification, iodoacetamide (IAA) was added at a working concentration of $20 \mathrm{mM}$ and lysates incubated for 1 hour at room temperature. In-solution digestion was carried out using SP3 beads and LysC (overnight incubation at $37^{\circ} \mathrm{C}$ ) at a concentration of 1 $\mu \mathrm{g}$ LysC (Promega) per $100 \mu \mathrm{g}$ protein followed by trypsin (Promega) digestion, also at $1 \mu \mathrm{g}$ enzyme per $100 \mu \mathrm{g}$ protein overnight at $37^{\circ} \mathrm{C}$. Peptides were eluted using acetonitrile (ACN) and quantified using a 3-(4-carboxybenzoyl)quinoline-2-carboxaldehyde (CBQCA) protein quantitation kit (Molecular Probes). Samples were diluted to a concentration of $1 \mu \mathrm{g} / 15 \mu \mathrm{L}$ with $5 \%$ formic acid and $1 \mu \mathrm{g}$ peptide was submitted for fractionation. Samples were fractionated using strong anion exchange chromatography, analyzed in an LTQ-Orbitrap Velos MS, and raw data processed using MaxQuant software (53). Subsequent statistical analysis and figure generation was carried out using Perseus software (17), Microsoft Excel, and GraphPad Prism. The mass spectrometry proteomics data have been deposited to the ProteomeXchange Consortium via the PRIDE (54) partner repository with the data set identifier PXD013672.

\section{Neutrophil protein lysates}

Isolated neutrophils were washed twice with cold PBS following culture or purification. Cells were pelleted by centrifugation at $300 \mathrm{~g}$ for 5 minutes and the supernatant removed. The pellet was resuspended in Laemmli buffer with PMSF, phosphatase inhibitors, and protease inhibitors $(100 \mu \mathrm{L}$ Laemmli buffer per 1 million murine or 10 million human neutrophils). An equal volume of 2 times SDS lysis buffer was added and mixed by pipetting. The sample was then boiled for 5 minutes prior to storage at $-80^{\circ} \mathrm{C}$ until use.

\section{Immunoblot for protein expression}

Neutrophil protein lysates were analyzed for protein expression by Western blot. Proteins were separated by SDS-PAGE (8\%, 10\%, or $12 \%$ gels depending on protein size) using the BioRad mini-protean system prior to wet transfer onto PVDF membrane (Merck Millipore). Membranes were blocked using 5\% skimmed milk powder for a minimum of 2 hours. Primary antibodies were from Cell Signaling Technology (p38 9212, P70 S6 kinase 2708, phospho-p70 S6 kinase 9234, HRP-linked anti-rat immunoglobulin 7077, HRP linked anti-mouse immunoglobulin 7076), Abcam (elastase Ab205670, MMP9 Ab58803, MPO Ab 188211), and Dako (HRP linked anti-rabbit immunoglobulin, p044801) and were made up in 5\% skimmed milk in 1× TBS-Tween (TBST, $0.05 \%$ Tween). Membranes were incubated at $4^{\circ} \mathrm{C}$ overnight on a rolling platform. Membranes underwent 3 washes in $1 \times$ TBST prior to incubation with the appropriate horseradish peroxidase-conjugated (HRP-conjugated) secondary antibody for 1 hour at room temperature. The membrane was washed as above prior to developing in enhanced chemiluminescent (ECL) detection reagent (GE Healthcare). Chemiluminescence was assessed using a Licor Odyssey Fc machine and quantified using ImageStudioLite software. Protein quantification was normalized to a loading control as follows: the membrane was stripped using Restore Western Blot stripping buffer (Thermo Fisher Scientific), washed with $\mathrm{dH}_{2} \mathrm{O}$, and then reprobed for the loading control protein (or, for the nonphosphorylated form of the protein of interest where appropriate) with primary and secondary antibodies as above. The membrane was then developed and protein quantified as above.

\section{Neutrophil RNA extraction and TaqMan analysis of gene expression} Percoll purified neutrophils were used to generate mRNA samples using the mirVana miRNA isolation kit (Invitrogen) as per manufacturer instructions followed by DNase treatment using the Invitrogen TURBO DNA free kit. The quantity and purity of the RNA were assessed using a Nanodrop 100 spectrophotometer. cDNA was generated using AMV reverse transcriptase and random primers (Promega) and samples were run on a Techne Thermal cycler as follows: $23^{\circ} \mathrm{C}$ for 5 minutes, $42^{\circ} \mathrm{C}$ for 2 hours, $99^{\circ} \mathrm{C}$ for 2 minutes. Samples were stored at $-20^{\circ} \mathrm{C}$ until use.

Gene expression was analyzed using predesigned qPCR primer/probe assays from Integrated DNA Technologies (IDT) (Elane Mm.PT.58.6682392.gs, MMP9 Mm.PT.58.10100097, and MPO Mm.PT.58.5251395) and Prime Time Gene Expression Mastermix 
(IDT). For all assays, samples were run in triplicate and the gene of interest expressed relative to expression of a housekeeping gene (beta-actin, Mm.PT.39a.22214843.g). Assays were run on a 7900HT Fast real-time PCR system (Applied Biosystems). Data was analyzed using SDS 2.0 software (Thermo Fisher Scientific).

\section{Analysis of BAL supernatant}

The first $0.8 \mathrm{~mL}$ aliquot of BAL fluid was used to analyze the activity or quantity of a number of proteins and metabolites within the BAL fluid. The freshly isolated aliquot was kept on ice and centrifuged at $350 \mathrm{~g}$ for 10 minutes at $4^{\circ} \mathrm{C}$ to pellet the BAL leukocytes and erythrocytes present in the aliquot. The supernatant was removed into a fresh tube, flash frozen on dry ice, and stored at $-80^{\circ} \mathrm{C}$ until use. In all assays, commercial kits were used as per the manufacturer protocol (IgM ELISA Abcam Ab133047, Albumin ELISA Abcam Ab108792, EnzCheck Elastase Activity Assay kit Invitrogen E12056, EnzCheck MPO Activity Assay kit Invitrogen E33856, MMP9 ELISA Bio-Techne MMPT90) and the optimal dilution of samples was preoptimized for each different analyte.

\section{Analysis of murine neutrophils by flow cytometry}

Blood (300-500 $\mu \mathrm{L}$ ) was collected into $30 \mu \mathrm{L}$ EDTA and underwent red cell lysis as described above. Cells were counted using a hemocytometer and $2 \times 10^{5}$ cells were stained for flow cytometric analysis. Whole BAL was counted by hemocytometer and resuspended at a density of $1 \times 10^{6}$ cells $/ \mathrm{mL}$. An aliquot of $2 \times 10^{5}$ cells was then stained for analysis. Following digest as described above, $2 \times 10^{5}$ cells were stained and analyzed by flow cytometry.

\section{Murine flow cytometry staining protocol}

A viability assay was conducted for lung digest samples only. Samples were first resuspended in Live/Dead fixable Aqua stain (Invitrogen) as per manufacturer's protocol. For all tissue types, samples were blocked in $25 \mu \mathrm{L}$ PBS with $10 \%$ mouse serum and 1\% Fc Block (Biolegend) for 15 minutes on ice. The antibody master mix was made up in PBS and $50 \mu \mathrm{L}$ master mix was added to each sample. Cells were stained for 30 minutes on ice in the dark prior to being washed 3 times in FACS buffer and either being fixed in 4\% PFA for 15 minutes at room temperature or analyzed immediately on a BD LSR Fortessa cell analyzer. For all flow cytometry experiments, data were acquired using FACS Diva software and analyzed using FlowJo software (version 10.1). Flow cytometry antibodies are detailed in Supplemental Table 6.

\section{Dextran and albumin uptake assays}

Neutrophils were preincubated in normoxia or hypoxia, in glucosedeplete or -replete media for 2 hours prior to the addition of Texas red-labeled $70 \mathrm{kDa}$ dextran (Invitrogen), FITC, or DQ-Greenlabeled BSA (Invitrogen) with or without LPS (100 ng/mL). E64 (2 $\mu \mathrm{M}$, Sigma-Aldrich), chloroquine (10 $\mu \mathrm{M}$, Sigma-Aldrich), rapamycin (50 nM, Sigma-Aldrich), or the activator of mTOR MHY1485 $(2 \mu \mathrm{M}$, Merck Chemicals) were added for 1 hour prior to fluorescent albumin. Uptake was quantified by flow cytometry and localization assessed by confocal microscopy.

\section{Confocal microscopy}

Confocal microscopy was used to delineate the intracellular location of the fluorescently labeled compounds described above. Neutrophils were washed and fixed in $4 \%$ PFA prior to permeabilization with $0.1 \%$
Triton X-100. The cells were washed again in PBS before staining with a deep-red cell mask ( $1 \mu \mathrm{g} / \mathrm{mL}$, Invitrogen). For LAMP1 colocalization, cells were fixed using cytofix/cytoperm (BD Biosciences) and stained (LAMP1, Abcam mouse monoclonal) in 1× Perm/Wash buffer (containing FCS and saponin). Alexa Fluor 647-conjugated goat anti-mouse secondary antibody (Abcam) was used for detection, with pellets resuspended in ProLong Gold liquid mountant with DAPI for nuclear staining (Invitrogen). Confocal images were acquired using a Leica SP5 confocal laser scanning microscope with Leica Application Suite software.

\section{Statistics}

Graphs were created in GraphPad Prism 7, Microsoft Excel, or Perseus (17). KEGG pathway enrichment analysis (19) was carried out using online DAVID bioinformatics tools. A $P$ value less than 0.05 was considered significant.

Study approval. C57BL/6JOlA mice were purchased from Envigo. Male mice aged 8-10 weeks were used in all experiments. All mouse experiments were conducted in accordance with the Home Office Animals (Scientific Procedures) Act of 1986 under a Home Office Project License (PPL 70/8364) with local ethics approval.

Human peripheral venous blood was taken from healthy volunteers with written informed consent as approved by the University of Edinburgh Centre for Inflammation Research Blood Resource Management Committee (AMREC 15-HV-013). Volunteers were 61\% female and aged $21-40$ years ( $55 \%$ in the $21-30$ years age bracket and $65 \%$ in the $31-40$ years age bracket).

The mass spectrometry proteomics data have been deposited to the ProteomeXchange Consortium via the PRIDE (54) partner repository with the data set identifier PXD013672.

\section{Author contributions}

ERW performed experimental design, all experiments, and data acquisition and interpretation. AJMH and JH performed proteomic data acquisition and interpretation. AVK performed amino acid tracing data acquisition and interpretation. $\mathrm{BG}$ and $\mathrm{AF}$ performed experimental design for metabolite data set acquisition. TM and PS performed data acquisition and interpretation of metabolic data sets. PC, FM, ASM, DCH, TMP, and RSD performed in vivo data acquisition. WV and GRB provided metabolic intermediary data acquisition. RG and EMR assisted with in vitro assays. DAC performed experimental design and conducted scientific direction. MKW conducted scientific direction and wrote the manuscript. SRW performed experimental design and data analysis, conducted scientific direction, and wrote the manuscript.

\section{Acknowledgments}

We are grateful to the MS facility at the College of Life Sciences of the University of Dundee and to the Mass Spectrometry and Proteomic Facilities at the Institute for Genetics and Molecular Medicine at the University of Edinburgh. We would like to thank the Flow Cytometry and Cell Sorting facility at the Queens Medical Research Institute, University of Edinburgh, for their advice and assistance. We are very grateful to Trevor Hansel and Ryan Thwaites (Imperial College, London, United Kingdom) for generously providing us with the filter cards for measurement of airway metabolites and for sharing their expertise to help with this work. Finally, we are grateful to the staff in the Bioresearch and Veterinary Services 
department at the Little France site, University of Edinburgh, for all of their assistance with the animal work in this manuscript. This work was supported by Wellcome Trust Clinical Fellowship awards (108717/Z/15/Z ERW, 098516, and 209220 to SRW).
Address correspondence to: Sarah R. Walmsley, University of Edinburgh Centre for Inflammation Research, Queen's Medical Research Institute, University of Edinburgh, Edinburgh EH16 4TJ, United Kingdom. Phone: 0131.242.6785; Email: sarah.walmsley@ed.ac.uk.
1. Walmsley SR, et al. Hypoxia-induced neutrophil survival is mediated by HIF-1alpha-dependent NFkappaB activity. JExp Med. 2005;201(1):105-115.

2. Mecklenburgh KI, et al. Involvement of a ferroprotein sensor in hypoxia-mediated inhibition of neutrophil apoptosis. Blood. 2002;100(8):3008-3016.

3. Hannah S, et al. Hypoxia prolongs neutrophil survival in vitro. FEBS Lett. 1995;372(2-3):233-237.

4. Majumdar SR, et al. Oxygen saturations less than $92 \%$ are associated with major adverse events in outpatients with pneumonia: a population-based cohort study. Clin Infect Dis. 2011;52(3):325-331.

5. Sanz F, et al. Hypoxemia adds to the CURB-65 pneumonia severity score in hospitalized patients with mild pneumonia. Respir Care. 2011;56(5):612-618.

6. Thompson AAR, et al. Hypoxia determines survival outcomes of bacterial infection through HIF1alpha dependent re-programming of leukocyte metabolism. Sci Immunol. 2017;2(8):eaal2861.

7. Sadiku P, et al. Prolyl hydroxylase 2 inactivation enhances glycogen storage and promotes excessive neutrophilic responses. JClin Invest. 2017;127(9):3407-3420.

8. Zemans RL, Matthay MA. What drives neutrophils to the alveoli in ARDS? Thorax. 2017;72(1):1-3.

9. Flick MR, et al. Leukocytes are required for increased lung microvascular permeability after microembolization in sheep. Circ Res. 1981;48(3):344-351.

10. Bachofen M, Weibel ER. Alterations of the gas exchange apparatus in adult respiratory insufficiency associated with septicemia. Am Rev Respir Dis. 1977;116(4):589-615.

11. Dreyfuss D, Saumon G. Role of tidal volume, FRC, and end-inspiratory volume in the development of pulmonary edema following mechanical ventilation. Am Rev Respir Dis. 1993;148(5):1194-1203.

12. Acute Respiratory Distress Syndrome Network, et al. Ventilation with lower tidal volumes as compared with traditional tidal volumes for acute lung injury and the acute respiratory distress syndrome. $N$ EnglJ Med.2000;342(18):1301-1308.

13. Dorward DA, et al. Tissue-specific immunopathology in fatal COVID-19. Am J Respir Crit Care Med. 2021;203(2):192-201.

14. Grommes J, Soehnlein O. Contribution of neutrophils to acute lung injury. MolMed. 2011;17(3-4):293-307.

15. Cowland JB, Borregaard N. Granulopoiesis and granules of human neutrophils. Immunol Rev. 2016;273(1):11-28.

16. Borregaard N, et al. Biosynthesis of granule proteins in normal human bone marrow cells. Gelatinase is a marker of terminal neutrophil differentiation. Blood. 1995;85(3):812-817.

17. Tyanova S, et al. The Perseus computational platform for comprehensive analysis of (prote)omics data. Nat Methods. 2016;13(9):731-740.

18. Wiśniewski JR, et al. A "Proteomic Ruler" for protein copy number and concentration estimation without spike-in standards. Mol Cell Proteomics. 2014;13(12):3497-3506.

19. Huang DW, et al. Bioinformatics enrichment tools: paths toward the comprehensive functional analysis of large gene lists. Nucleic Acids Res. 2009;37(1):1-13.

20. Huang DW, et al. Systematic and integrative analysis of large gene lists using DAVID bioinformatics resources. Nat Protoc. 2009;4(1):44-57.

21. Dorward DA, et al. The role of formylated peptides and formyl peptide receptor 1 in governing neutrophil function during acute inflammation. Am J Pathol.2015;185(5):1172-1184.

22. Silveira AAA, et al. TNF induces neutrophil adhesion via formin-dependent cytoskeletal reorganization and activation of $\beta$-integrin function.

JLeukoc Biol. 2018;103(1):87-98.

23. McLeish KR, et al. Frontline science: tumor necrosis factor- $\alpha$ stimulation and priming of human neutrophil granule exocytosis. JLeukoc Biol. 2017;102(1):19-29.

24. Murray J, et al. Regulation of neutrophil apoptosis by tumor necrosis factor-alpha: requirement for TNFR55 and TNFR75 for induction of apoptosis in vitro. Blood.1997;90(7):2772-2783.

25. Ramadass M, et al. Rab27a regulates GM-CSFdependent priming of neutrophil exocytosis. J Leukoc Biol. 2017;101(3):693-702.

26. Kobayashi SD, et al. Spontaneous neutrophil apoptosis and regulation of cell survival by granulocyte macrophage-colony stimulating factor. JLeukoc Biol. 2005;78(6):1408-1418.

27. Faurschou M, Borregaard N. Neutrophil granules and secretory vesicles in inflammation. Microbes Infect. 2003;5(14):1317-1327.

28. Garnett JP, et al. Proinflammatory mediators disrupt glucose homeostasis in airway surface liquid. Jimmunol. 2012;189(1):373-380.

29. Mallia $P$, et al. Role of airway glucose in bacterial infections in patients with chronic obstructive pulmonary disease. JAllergy Clin Immunol. 2018;142(3):815-823.

30. Bhutia YD, Ganapathy V. Glutamine transporters in mammalian cells and their functions in physiology and cancer. Biochim Biophys Acta. 2016;1863(10):2531-2539.

31. Commisso C, et al. Macropinocytosis of protein is an amino acid supply route in Ras-transformed cells. Nature. 2013;497(7451):633-637.

32. Palm W, et al. The utilization of extracellular proteins as nutrients is suppressed by mTORC1. Cell. 2015;162(2):259-270.

33. Davidson SM, et al. Direct evidence for cancercell-autonomous extracellular protein catabolism in pancreatic tumors. Nat Med.2017;23(2):235-241.

34. Sadiku P, et al. Neutrophils fuel effective immune responses through gluconeogenesis and glycogenesis. Cell Metab. 2021;33(2):411-423.

35. Recouvreux MV, Commisso C. Macropinocytosis: a metabolic adaptation to nutrient stress in cancer. Front Endocrinol (Lausanne). 2017;8:261.

36. Canton J, et al. Calcium-sensing receptors signal constitutive macropinocytosis and facilitate the uptake of NOD2 ligands in macrophages. Nat Commun. 2016;7(1):11284.
37. Sancak Y, et al. Ragulator-Rag complex targets mTORC1 to the lysosomal surface and is necessary for its activation by amino acids. Cell. 2010;141(2):290-303.

38. Choi YJ, et al. Inhibitory effect of mTOR activator MHY1485 on autophagy: suppression of lysosomal fusion. PLoS One. 2012;7(8):e43418.

39. Schröder BA, et al. The proteome of lysosomes. Proteomics. 2010;10(22):4053-4076.

40. Wibo M, Poole B. Protein degradation in cultured cells. II. The uptake of chloroquine by rat fibroblasts and the inhibition of cellular protein degradation and cathepsin B1.JCell Biol. 1974;63(2 Pt 1):430-440.

41. Neeley SP, et al. Selective regulation of expression of surface adhesion molecules Mac-1, L-selectin, and VLA-4 on human eosinophils and neutrophils. Am J Respir Cell Mol Biol.1993;8(6):633-639.

42. in 't Veen JC, et al. CD11b and L-selectin expression on eosinophils and neutrophils in blood and induced sputum of patients with asthma compared with normal subjects. Clin Exp Allergy. 1998;28(5):606-615.

43. Shen $\mathrm{H}$, et al. Chloroquine attenuates paraquat-induced lung injury in mice by altering inflammation, oxidative stress and fibrosis. Int Immunopharmacol. 2017;46:16-22.

44. Zhang L, et al. Chloroquine relieves acute lung injury in rats with acute hemorrhagic necrotizing pancreatitis. JHuazhong Univ Sci Technolog Med Sci. 2013;33(3):357-360.

45. Hidalgo A, et al. The neutrophil life cycle. Trends Immunol. 2019;40(7):584-597.

46. Jones HA, et al. In vivo measurement of neutrophil activity in experimental lung inflammation. AmJ Respir Crit Care Med.1994;149(6):1635-1639.

47. Eales KL, et al. Hypoxia and metabolic adaptation of cancer cells. Oncogenesis. 2016;5(1):e190.

48. Hays RC, Mandell GL. PO2, pH, and redox potential of experimental abscesses. Proc Soc Exp Biol Med. 1974;147(1):29-30.

49. Campbell EL, et al. Transmigrating neutrophils shape the mucosal microenvironment through localized oxygen depletion to influence resolution of inflammation. Immunity. 2014;40(1):66-77.

50. Ng CT, et al. Synovial tissue hypoxia and inflammation in vivo. Ann Rheum Dis. 2010;69(7):1389-1395

51. Harris AJ, et al. IL4R $\alpha$ signaling abrogates hypoxic neutrophil survival and limits acute lung injury responses in vivo. Am J Respir Crit Care Med. 2019;200(2):235-246.

52. Walmsley SR, et al. Prolyl hydroxylase 3 (PHD3) is essential for hypoxic regulation of neutrophilic inflammation in humans and mice. JClin Invest. 2011;121(3):1053-1063.

53. Cox J, Mann M. MaxQuant enables high peptide identification rates, individualized p.p.b.-range mass accuracies and proteome-wide protein quantification. Nat Biotechnol. 2008;26(12):1367-1372.

54. Perez-Riverol Y, et al. The PRIDE database and related tools and resources in 2019: improving support for quantification data. Nucleic Acids Res. 2019;47(D1):D442-D450. 